Discussiones Mathematicae

Differential Inclusions, Control and Optimization 34 (2014) 131-147

doi:10.7151/dmdico.1155

\title{
PROPERTIES OF GENERALIZED SET-VALUED STOCHASTIC INTEGRALS
}

\author{
Michą KisielewicZ \\ Faculty of Mathematics, Computer Science and Econometrics \\ University of Zielona Góra \\ Prof. Z. Szafrana 4a, 65-516 Zielona Góra \\ e-mail: M.Kisielewicz@wmie.uz.zgora.pl
}

\begin{abstract}
The paper is devoted to properties of generalized set-valued stochastic integrals defined in [10]. These integrals generalize set-valued stochastic integrals defined by E.J. Jung and J.H. Kim in the paper [4]. Up to now we were not able to construct any example of set-valued stochastic processes, different on a singleton, having integrably bounded set-valued integrals defined in [4]. It was shown by M. Michta (see [11]) that in the general case set-valued stochastic integrals defined by E.J. Jung and J.H. Kim, are not integrably bounded. Generalized set-valued stochastic integrals, considered in the paper, are in some non-trivial cases square integrably bounded and can be applied in the theory of stochastic differential equations with set-valued solutions.
\end{abstract}

Keywords: set-valued mappings, set-valued integrals, set-valued stochastic processes.

2010 Mathematics Subject Classification: 60H05, 28B20, $47 \mathrm{H} 04$.

\section{INTRODUCTION}

The paper is devoted to properties of generalized set-valued stochastic integrals, defined in the author paper [10] for a nonempty subsets of the space $\mathbb{L}^{2}\left(\mathbb{R}^{+} \times \Omega, \Sigma_{\mathbb{F}}, \mathbb{R}^{d \times m}\right)$ of all square integrable $\mathbb{F}$-nonanticipative matrix-valued processes. For a given $m$-dimensional $\mathbb{F}$-Brownian motion $B=\left(B_{t}\right)_{t \geq 0}$ defined on a filtered probability space $\mathcal{P}_{\mathbb{F}}=(\Omega, \mathcal{F}, \mathbb{F}, P)$ and a nonempty subset $\mathcal{G}$ of the space $\mathbb{L}^{2}\left(\mathbb{R}^{+} \times \Omega, \Sigma_{\mathbb{F}}, \mathbb{R}^{d \times m}\right)$, a generalized set-valued stochastic integral $\int_{0}^{t} \mathcal{G} d B_{\tau}$ is understood as an $\mathcal{F}_{t}$-measurable set-valued random variable 
with values in the $d$-dimensional Euclidean space $\mathbb{R}^{d}$ and subtrajectory integrals $S_{\mathcal{F}_{t}}\left(\int_{0}^{t} \mathcal{G} d B_{\tau}\right)$ equal to $\overline{\operatorname{dec}} \mathcal{J}_{t}(\mathcal{G})$. By $\mathcal{J}_{t}$ we denote the Itô isometry defined on the space $\mathbb{L}^{2}\left(\mathbb{R}^{+} \times \Omega, \Sigma_{\mathbb{F}}, \mathbb{R}^{d \times m}\right)$ by setting $\mathcal{J}_{t}(g)=\int_{0}^{t} g_{\tau} d B_{\tau}$ for every $g \in \mathbb{L}^{2}\left(\mathbb{R}^{+} \times \Omega, \Sigma_{\mathbb{F}}, \mathbb{R}^{d \times m}\right)$. Subtrajectory integrals $S_{\mathcal{F}_{t}}\left(\int_{0}^{t} \mathcal{G} d B_{\tau}\right)$ of $\int_{0}^{t} \mathcal{G} d B_{\tau}$ is defined as a set of all $\mathcal{F}_{t}$-measurable and square integrable selectors of $\int_{0}^{t} \mathcal{G} d B_{\tau}$. It will be also denoted by $S_{t}\left(\int_{0}^{t} \mathcal{G} d B_{\tau}\right)$. In particular, if $\mathcal{G}$ is a nonempty decomposable subset of $\mathbb{L}^{2}\left(\mathbb{R}^{+} \times \Omega, \Sigma_{\mathbb{F}}, \mathbb{R}^{d \times m}\right)$ then $\int_{0}^{t} \mathcal{G} d B_{\tau}=\int_{0}^{t} G_{\tau} d B_{\tau}$, where $G=$ $\left(G_{t}\right)_{t \geq 0}$ is an $\mathbb{F}$-nonanticipative set-valued process such that $S_{\mathbb{F}}(G)=\operatorname{cl}_{\mathbb{L}}(\mathcal{G})$, where $S_{\mathbb{F}}(G)=\left\{g \in \mathbb{L}^{2}\left(\mathbb{R}^{+} \times \Omega, \Sigma_{\mathbb{F}}, \mathbb{R}^{d \times m}\right): g_{t}(\omega) \in G_{t}(\omega)\right.$ for a.e. $(t, \omega) \in$ $\left.\mathbb{R}^{+} \times \Omega\right\}$. Set-valued stochastic integrals of the form $\int_{0}^{t} G_{\tau} d B_{\tau}$ have been defined by E.J. Jung and J.H. Kim in the paper [4], basing on the definition of set-valued functional stochastic integrals defined in the author papers [5] and [6] (see also [8]).

The generalized set-valued stochastic integrals, presented in this paper have better properties than set-valued stochastic integrals defined in the paper [4]. In particular, they are integrably bounded for some subsets of the space $\mathbb{L}^{2}\left(\mathbb{R}^{+} \times\right.$ $\left.\Omega, \Sigma_{\mathbb{F}}, \mathbb{R}^{d \times m}\right)$. Integrable boundedness of set-valued stochastic integrals, defined by E.J. Jung and J.H. Kim, has been investigated by the author of the present paper (see [7-9]) without positive results. We were not able to present any example of multifunction, different on a singleton, having integrably bounded setvalued integral defined in [4]. Unfortunately, the result dealing with integrable boundedness of multifunctions with finite representations Castaing, presented in [9] is not true. The problem was also considered by M. Michta, who has showed (see [11]) that in the general case set-valued integrals, defined by E.J. Jung and J.H. Kim, are not integrably bounded.

Apart from the extension of the definition of set-valued stochastic integrals, we shall also extend the definition of the set-valued conditional expectation. It will be defined for every nonempty subsets of the space $\mathbb{L}\left(\Omega, \mathcal{F}, P, \mathbb{R}^{d}\right)$ and called a generalized set-valued conditional expectation. The present paper is organized as follows. Section 2 contains basic notions of the theory of set-valued stochastic processes, whereas Section 3 is devoted to properties of generalized set-valued stochastic integrals. Integrable boundedness of generalized set-valued stochastic integrals is considered in Section 4. Basic properties of indefinite generalized set-valued stochastic integrals are contained in the last Section of the paper.

Let $(X, \rho)$ be a metric space and denote by $\mathrm{Cl}(X)$ a space of all nonempty closed subsets of $X$. For every $A, C \in \mathrm{Cl}(X)$ let $\bar{h}(A, C)=\sup \{d(a, C): a \in A\}$, where $d(a, C)=\inf \{\rho(a, c): c \in C\}$. The Hausdorff distance $h(A, C)$ between $A, C \in \mathrm{Cl}(X)$ is defined by $h(A, C)=\max \{\bar{h}(A, C), \bar{h}(C, A)\}$. Given a sequence $\left(A_{n}\right)_{n \geq 1} \subset \operatorname{Cl}(X) \cup\{\emptyset\}$ by $\underline{\operatorname{Lim}} A_{n}$ and $\overline{\operatorname{Lim}} A_{n}$ we denote its Kuratowski limits inferior and superior defined by $\underline{\operatorname{Lim}} A_{n}=\left\{x \in X: \lim d\left(x, A_{n}\right)=0\right\}$ and 
$\overline{\operatorname{Lim}} A_{n}=\left\{x \in X: \underline{\lim } d\left(x, A_{n}\right)=0\right\}$, respectively. It can be verified (see [2, 3]) that $\underline{\operatorname{Lim}} A_{n}=\left\{x \in X: x=\lim x_{n}, x_{n} \in A_{n}, n \geq 1\right\}$ and $\overline{\operatorname{Lim}} A_{n}=\{x \in X:$ $\left.x=\lim x_{n_{k}}, x_{n_{k}} \in A_{n_{k}}, n_{1}<n_{2}<\cdots<n_{k}<\cdots\right\}$. Immediately from the above definions we get $\underline{\operatorname{Lim}} A_{n} \subset \overline{\operatorname{Lim}} A_{n}$. We call a sequence $\left(A_{n}\right)_{n \geq 1}$ convergent in the Kuratowski sense to $A \in C l(X) \cup\{\emptyset\}$ if $A=\underline{\operatorname{Lim}} A_{n}=\overline{\operatorname{Lim}} A_{n}$. The limit $A$ is denoted by $\operatorname{Lim} A_{n}$ and said to be Kuratowski's limit of a sequence $\left(A_{n}\right)_{n \geq 1}$. If $A_{1} \subset A_{2} \subset A_{3} \subset \ldots$, then a sequence $\left(A_{n}\right)_{n \geq 1}$ is convergent in the Kuratowski sense and $\operatorname{Lim} A_{n}=\overline{\bigcup_{n \geq 1} A_{n}}$.

\section{Set-Valued Stochastic PROCESSES}

Throughout the paper we shall deal with a complete filtered probability space $\mathcal{P}_{\mathbb{F}}=(\Omega, \mathcal{F}, \mathbb{F}, P)$ with a filtration $\mathbb{F}=\left(\mathcal{F}_{t}\right)_{t \geq 0}$ satisfying the usual conditions. By an $r$-dimensional set-valued random variable we mean a closed valued $\mathcal{F}$ measurable multifunction, i.e., a multifunction $\mathcal{Z}: \Omega \rightarrow \mathrm{Cl}\left(\mathbb{R}^{r}\right)$ such that $\{\omega \in$ $\Omega: \mathcal{Z}(\omega) \cap C \neq \emptyset\} \in \mathcal{F}$ for every $C \in \mathrm{Cl}\left(\mathbb{R}^{r}\right)$. A family $G=\left(G_{t}\right)_{t \geq 0}$ of setvalued random variables $G_{t}: \Omega \rightarrow \mathrm{Cl}\left(\mathbb{R}^{r}\right)$ is said to be a set-valued stochastic process defined on $\mathcal{P}_{\mathbb{F}}$. Similarly as in the theory of point valued stochastic processes, a set-valued stochastic process can be defined as a multifunction $G$ : $\mathbb{R}^{+} \times \Omega \rightarrow \mathrm{Cl}\left(\mathbb{R}^{r}\right)$ such that $G(t, \cdot)$ is a set-valued random variable for every $t \geq 0$. Such defined stochastic process is said to be $\mathbb{F}$-nonanticipative if $G$ is $\beta\left(\mathbb{R}^{+}\right) \otimes \mathcal{F}$-measurable and $G(t, \cdot)$ is $\mathcal{F}_{t}$-measurable for every $t \geq 0$, where $\beta\left(\mathbb{R}^{+}\right)$denotes the Borel $\sigma$-algebra on $\mathbb{R}^{+}$. It is easy to see that the set-valued process $G$ is $\mathbb{F}$-nonanticipative if and only if the multifunction $G: \mathbb{R}^{+} \times \Omega \rightarrow$ $\mathrm{Cl}\left(\mathbb{R}^{r}\right)$ is $\Sigma_{\mathbb{F}^{-}}$-measurable, where $\Sigma_{\mathbb{F}}$ is a $\sigma$-algebra on $\mathbb{R}^{+} \times \Omega$ defined by $\Sigma_{\mathbb{F}}=$ $\left\{A \in \beta\left(\mathbb{R}^{+}\right) \otimes \mathcal{F}: A^{t} \in \mathcal{F}_{t}\right.$ for $\left.t \geq 0\right\}$, where $A^{t}$ denotes the section of a set $A$ at $t \in \mathbb{R}^{+}$. For a given $\mathbb{F}$-nonanticipative set-valued stochastic process $G=\left(G_{t}\right)_{t \geq 0}$ with values in $\mathrm{Cl}\left(\mathbb{R}^{d \times m}\right)$ defined on a filtered probability space $\mathcal{P}_{\mathbb{F}}=(\Omega, \mathcal{F}, \mathbb{F}, P)$, its subtrajectory integrals $S_{\mathbb{F}}(G)$ is defined by $S_{\mathbb{F}}(G)=\{g \in$ $\mathbb{L}^{2}\left(\mathbb{R}^{+} \times \Omega, \Sigma_{\mathbb{F}}, \mathbb{R}^{d \times m}\right): g_{t}(\omega) \in G_{t}(\omega)$ for a.e. $\left.(t, \omega) \in \mathbb{R}^{+} \times \Omega\right\}$. If $S_{\mathbb{F}}(G) \neq \emptyset$ then $G$ is said to be Itô integrable.

Properties of set-valued random variables and set-valued stochastic processes follow immediately from properties of measurable multifunctions (see [1] and [2]). For a given separable Banach space $\mathcal{X}$ and a $\sigma$-finite complete measure space $(\mathbb{T}, \mathcal{A}, \mu)$, a multifunction $\mathcal{Z}: \mathbb{T} \rightarrow \operatorname{Cl}(\mathcal{X})$ is said to be Aumann integrable if its subtrajectory integrals, denoted by $S_{\mathcal{A}}(\mathcal{Z})$ or simply by $S(\mathcal{Z})$ is nonempty. It can be proved (see [1]) that an Aumann integrable multifunction $\mathcal{Z}$ is square integrably bounded if and only if $S(\mathcal{Z})$ is a bounded subset of $\mathbb{L}^{2}(\mathbb{T}, \mathcal{A}, \mathcal{X})$. It can be verified (see [3], Corollary 3.5 of Chap. 2) that if $\mathcal{Z}$ and $G$ are Aumann integrable and $S(\mathcal{Z})=S(G)$ then $\mathcal{Z}(t)=G(t)$ for $\mu$-a.e. $t \in \mathbb{T}$. It is clear that 
$S(\mathcal{Z})$ is a closed subset of $\mathbb{L}(\mathbb{T}, \mathcal{A}, \mathcal{X})$. It is also decomposable, i.e., for every $A \in \mathcal{A}$ and $u, v \in S(\mathcal{Z})$ one has $\mathbb{I}_{A} u+\mathbb{I}_{\mathbb{T} \backslash A} v \in S(\mathcal{Z})$. If $\mathcal{Z}$ is Aumann integrable then there is a Castaing representation $\left(z_{n}\right)_{n=1}^{\infty}$ of $\mathcal{Z}$ such that $\left(z_{n}\right)_{n=1}^{\infty} \subset S(\mathcal{Z})$, and therefore (see [1], Lemma 1.3), for every $z \in S(\mathcal{Z})$ and $\varepsilon>0$ there exist a finite $\mathcal{A}$-measurable partition $\left(A_{k}\right)_{k=1}^{N}$ of $\mathbb{T}$ and a family $\left(z_{n_{k}}\right)_{k=1}^{N} \subset\left\{z_{n}: n \geq 1\right\}$ such that $\int_{\mathbb{T}}\left|z-\sum_{k=1}^{N} \mathbb{I}_{A_{k}} z_{n_{k}}\right|^{2} d \mu<\varepsilon$. In what follows the family of all finite $\mathcal{A}$-measurable partitions of $\mathbb{T}$ is denoted by $\Pi(\mathbb{T}, \mathcal{A})$. For a given $\Lambda \subset \mathbb{L}(\mathbb{T}, \mathcal{A}, \mathcal{X})$ by $\operatorname{dec}(\Lambda)$ we denote the decomposable hull of $\Lambda$, i.e., the smallest decomposable subset of $\mathbb{L}(\mathbb{T}, \mathcal{A}, \mathcal{X})$ containing $\Lambda$. In a similar way the closed decomposable hull $\overline{\operatorname{dec}}(\Lambda)$ is defined. It can be verified that $\overline{\operatorname{dec}}(\Lambda)=\operatorname{cl}_{\mathbb{L}}[\operatorname{dec}(\Lambda)]$, where the closure is taken in the norm topology of $\mathbb{L}(\mathbb{T}, \mathcal{A}, \mathcal{X})$.

Immediately from the above properties of Aumann integrable multifunctions it follows that if $\left(z_{n}\right)_{n=1}^{\infty} \subset S(\mathcal{Z})$ is a Castaing representation of a multifunction $\mathcal{Z}$, then $S(\mathcal{Z})=\overline{\operatorname{dec}}\left\{z_{n}: n \geq 1\right\}$. Indeed, it is clear that $\overline{\operatorname{dec}}\left\{z_{n}: n \geq 1\right\} \subset$ $S(\mathcal{Z})$. On the other hand, for every $z \in S(\mathcal{Z})$ and $\varepsilon>0$ there exist a partition $\left(A_{k}\right)_{k=1}^{N} \in \Pi(\mathbb{T}, \mathcal{A})$ and a family $\left(z_{n_{k}}\right)_{k=1}^{N} \subset\left\{z_{n}: n \geq 1\right\}$ such that $\int_{\mathbb{T}} \mid z-$ $\left.\sum_{k=1}^{N} \mathbb{I}_{A_{k}} z_{n_{k}}\right|^{2} d \mu \leq \varepsilon$, which implies that $z \in \overline{\operatorname{dec}}\left\{z_{n}: n \geq 1\right\}$. Thus $S(\mathcal{Z})=$ $\operatorname{dec}\left\{z_{n}: n \geq 1\right\}$. Finally, let us note (see [3], Th. 3.8 of Chap. 2) that a nonempty closed set $\mathcal{K} \subset \mathbb{L}^{2}(T, \mathcal{A}, \mathcal{X})$ is decomposable if and only if there exists an $\mathcal{A}$ measurable multifunction $F: T \rightarrow \mathrm{Cl}(\mathcal{X})$ such that $\mathcal{K}=S(F)$.

For a given integrably bounded set-valued random variable $\mathcal{Z}: \Omega \rightarrow \mathrm{Cl}\left(\mathbb{R}^{r}\right)$ and a $\sigma$-algebra $\mathcal{G} \subset \mathcal{F}$ there exists (see [1], Th. 5.1) a unique $\mathcal{G}$-measurable setvalued random variable $E[\mathcal{Z} \mid \mathcal{G}]: \Omega \rightarrow \mathrm{Cl}\left(\mathbb{R}^{r}\right)$ such that $S(E[\mathcal{Z} \mid \mathcal{G}])=\operatorname{cl}_{\mathbb{L}}\{E[f \mid \mathcal{G}]:$ $f \in S(\mathcal{Z})\}$, where the closure is taken in the norm topology of $\mathbb{L}\left(\Omega, \mathcal{F}, \mathbb{R}^{r}\right)$. A set-valued random variable $E[\mathcal{Z} \mid \mathcal{G}]$ is said to be the set-valued conditional expectation of $\mathcal{Z}$ relative to $\mathcal{G}$. We can extend the above definition to nonempty subsets of the space $\mathbb{L}\left(\Omega, \mathcal{F}, \mathbb{R}^{r}\right)$. Given a nonempty set $\Lambda \subset \mathbb{L}\left(\Omega, \mathcal{F}, \mathbb{R}^{r}\right)$ and a $\sigma$-algebra $\mathcal{G} \subset \mathcal{F}$ the generalized set-valued condition expectation $E[\Lambda \mid \mathcal{G}]$ of a set $\Lambda$ relative to $\mathcal{G}$ is defined to be an $\mathcal{G}$-measurable set-valued mapping $E[\Lambda \mid \mathcal{G}]: \Omega \rightarrow \operatorname{Cl}\left(\mathbb{R}^{r}\right)$ such that $S(E[\Lambda \mid \mathcal{G}])=\overline{\operatorname{dec}}_{\mathcal{G}}\{E[z \mid \mathcal{G}]: z \in \Lambda\}$, where the decomposable hull is taken with respect to a $\sigma$-algebra $\mathcal{G} \subset \mathcal{F}$. It is clear that if $\Lambda=S(\mathcal{Z})$, where $\mathcal{Z}$ is an Aumann integrable set-valued random variable, then $E[\Lambda \mid \mathcal{G}]=E[\mathcal{Z} \mid \mathcal{G}]$ a.s. Indeed, by the above definitions we get $S(E[S(\mathcal{Z}) \mid \mathcal{G}])=\overline{\operatorname{dec}}_{\mathcal{G}}\{E[z \mid \mathcal{G}]: z \in S(\mathcal{Z})\}=$ $\operatorname{cl}_{\mathbb{L}}\left[\operatorname{dec}_{\mathcal{G}}\{E[z \mid \mathcal{G}]: z \in S(\mathcal{Z})\}\right]=\operatorname{cl}_{\mathbb{L}}\{E[z \mid \mathcal{G}]: z \in S(\mathcal{Z})\}=S(E[\mathcal{Z} \mid \mathcal{G}])$, because the set $\{E[z \mid \mathcal{G}]: z \in S(\mathcal{Z})\}$ is decomposable with respect to the $\sigma$-algebra $\mathcal{G} \subset \mathcal{F}$. Therefore, $E[S(\mathcal{Z}) \mid \mathcal{G}]=E[\mathcal{Z} \mid \mathcal{G}]$ a.s.

\section{Some Properties of GENERALIZED SET-VALUED INTEGRALS}

We present here some general properties of generalized set-valued stochastic integrals. In what follows we shall assume that we have given an $m$-dimensional 
F-Brownian motion $B=\left(B_{t}\right)_{t \geq 0}$ defined on a complete filtered probability space $\mathcal{P}_{\mathbb{F}}$. Apart from subsets of the space $\mathbb{L}^{2}\left(\mathbb{R}^{+} \times \Omega, \Sigma_{\mathbb{F}}, \mathbb{R}^{d \times m}\right)$ we shall also consider subsets of the space $\mathbb{L}^{2}\left(\Omega, \mathcal{F}_{t}, \mathbb{R}^{d}\right)$ for every $t \geq 0$. The closures of subsets of both $\mathbb{L}^{2}\left(\mathbb{R}^{+} \times \Omega, \Sigma_{\mathbb{F}}, \mathbb{R}^{d \times m}\right)$ and $\mathbb{L}^{2}\left(\Omega, \mathcal{F}_{t}, \mathbb{R}^{d}\right)$ will be denoted by the same way by $\mathrm{cl}_{\mathbb{L}}$.

Lemma 3.1. For every nonempty set $\mathcal{G} \subset \mathbb{L}^{2}\left(\mathbb{R}^{+} \times \Omega, \Sigma_{\mathbb{F}}, \mathbb{R}^{d \times m}\right)$ and $t \geq 0$ one has

(i) $\mathcal{J}_{t}\left[\operatorname{cl}_{\mathbb{L}}(\mathcal{G})\right]=\operatorname{cl}_{\mathbb{L}}\left[\mathcal{J}_{t}(\mathcal{G})\right]$

(ii) $\mathcal{J}_{t}(\overline{\mathrm{co}}(\mathcal{G}))=\overline{\mathrm{co}} \mathcal{J}_{t}(\mathcal{G})$,

(iii) $\overline{\operatorname{dec}}\left\{\mathcal{J}_{t}[\overline{\mathrm{co}}(\mathcal{G})]\right\}=\overline{\mathrm{co}}\left[\overline{\operatorname{dec}} \mathcal{J}_{t}(\mathcal{G})\right]$,

(iv) if $(\Omega, \mathcal{F}, P)$ is a separable probability space then there is a sequence $\left(g^{n}\right)_{n=1}^{\infty} \subset$ $\mathcal{G}$ such that $\operatorname{cl}_{\mathbb{L}} \mathcal{J}_{t}(\mathcal{G})=\operatorname{cl}_{\mathbb{L}}\left\{\mathcal{J}_{t}\left(g^{n}\right): n \geq 1\right\}$ for every $t \geq 0$.

Proof. (i) By continuity of a mapping $\mathcal{J}_{t}$ one has $\mathcal{J}_{t}\left[\operatorname{cl}_{\mathbb{L}}(\mathcal{G})\right] \subset \operatorname{cl}_{\mathbb{L}}\left[\mathcal{J}_{t}(\mathcal{G})\right]$. For every $u \in \operatorname{cl}_{\mathbb{L}}\left[\mathcal{J}_{t}(\mathcal{G})\right]$ and every sequence $\left(g^{n}\right)_{n=1}^{\infty} \subset \mathcal{G}$ such that $E\left|u-\mathcal{J}_{t}\left(g^{n}\right)\right|^{2} \rightarrow 0$ we have $E\left|\mathcal{J}_{t}\left(g^{n}\right)-\mathcal{J}_{t}\left(g^{m}\right)\right|^{2} \rightarrow 0$ as $m, n \rightarrow \infty$. But $E\left|\mathcal{J}_{t}\left(g^{n}\right)-\mathcal{J}_{t}\left(g^{m}\right)\right|^{2}=$ $E \int_{0}^{t}\left|g_{\tau}^{n}-g_{\tau}^{m}\right|^{2} d \tau$ for every $t \geq 0$ and every $m, n \geq 1$. Then $\left(g^{n}\right)_{n=1}^{\infty}$ is a Cauchy sequence in the Banach space $\mathbb{L}^{2}\left(\mathbb{R}^{+} \times \Omega, \Sigma_{\mathbb{F}}, \mathbb{R}^{d \times m}\right)$. Thus there exists $g \in$ $\mathbb{L}^{2}\left(\mathbb{R}^{+} \times \Omega, \Sigma_{\mathbb{F}}, \mathbb{R}^{d \times m}\right)$ such that $E \int_{0}^{t}\left|g_{\tau}^{n}-g_{\tau}\right|^{2} d \tau \rightarrow 0$ as $n \rightarrow \infty$, which implies that $g \in \mathrm{cl}_{\mathbb{L}}(\mathcal{G})$ and $E\left|\mathcal{J}_{t}(g)-\mathcal{J}_{t}\left(g^{n}\right)\right|^{2} \rightarrow 0$ as $n \rightarrow \infty$. Then $\mathcal{J}_{t}(g) \in \mathcal{J}_{t}\left[\mathrm{cl}_{\mathbb{L}}(\mathcal{G})\right]$ because $\mathcal{J}_{t}\left[\operatorname{cl}_{\mathbb{L}}(\mathcal{G})\right]$ is a closed subset of the space $\mathbb{L}^{2}\left(\Omega, \mathcal{F}_{t}, \mathbb{R}^{d}\right)$. Hence it follows that $u \in \mathcal{J}_{t}\left[\operatorname{cl}_{\mathbb{L}}(\mathcal{G})\right]$ because $u=\mathcal{J}_{t}(g)$. Then $\operatorname{cl}_{\mathbb{L}}\left[\mathcal{J}_{t}(\mathcal{G})\right] \subset \mathcal{J}_{t}\left[\operatorname{cl}_{\mathbb{L}}(\mathcal{G})\right]$.

(ii) By linearity of a mapping $\mathcal{J}_{t}$ we have $\mathcal{J}_{t}(\operatorname{co} \mathcal{G})=\operatorname{co} \mathcal{J}_{t}(\mathcal{G})$, which implies that $\mathrm{cl}_{\mathbb{L}}\left[\mathcal{J}_{t}(\operatorname{co} \mathcal{G})\right]=\overline{\mathrm{co}} \mathcal{J}_{t}(\mathcal{G})$. Hence, by (i) it follows that $\mathcal{J}_{t}(\overline{\mathrm{co}}(\mathcal{G}))=\overline{\mathrm{co}}\left[\mathcal{J}_{t}(\mathcal{G})\right]$ because $\operatorname{cl}_{\mathbb{L}}\left[\mathcal{J}_{t}(\operatorname{co} \mathcal{G})\right]=\mathcal{J}_{t}(\overline{\operatorname{co}} \mathcal{G})$.

(iii) It is clear that $\left.\mathcal{J}_{t}[\overline{\mathrm{co}}(\mathcal{G})]\right\} \subset \overline{\mathrm{co}}\left[\overline{\operatorname{dec}} \mathcal{J}_{t}(\mathcal{G})\right]$ because $\mathcal{J}_{t}(\overline{\mathrm{co}}(\mathcal{G}))=\overline{\mathrm{co}} \mathcal{J}_{t}(\mathcal{G})$. Let us observe that $\overline{\mathrm{co}}\left[\overline{\mathrm{dec}} \mathcal{J}_{t}(\mathcal{G})\right]$ is a decomposable subset of the space $\mathbb{L}^{2}\left(\Omega, \mathcal{F}_{t}, \mathbb{R}^{d}\right)$. Indeed, by the properties of a set $\overline{\operatorname{dec}} \mathcal{J}_{t}(\mathcal{G})$ there is an $\mathcal{F}_{t}$-measurable set-valued random variable $F=\left(F_{t}\right)_{t \geq 0}$ with values in $\mathrm{Cl}\left(\mathbb{R}^{d}\right)$ and such that $\overline{\operatorname{dec}} \mathcal{J}_{t}(\mathcal{G})=S_{t}(F)$ for every $t \geq 0$. Then, $\overline{\operatorname{co}}\left[\overline{\operatorname{dec}} \mathcal{J}_{t}(\mathcal{G})\right]=\overline{\operatorname{co}} S_{t}(F)=S_{t}(\overline{\operatorname{co}} F)$, which implies that $\overline{\mathrm{co}}\left[\overline{\operatorname{dec}} \mathcal{J}_{t}(\mathcal{G})\right]$ is decomposable. Therefore, $\overline{\operatorname{dec}}\left\{\mathcal{J}_{t}[\overline{\operatorname{co}}(\mathcal{G})]\right\}$ $\subset \overline{\operatorname{co}}\left[\overline{\operatorname{dec}} \mathcal{J}_{t}(\mathcal{G})\right]$. On the other hand we have $\overline{\operatorname{dec}} \mathcal{J}_{t}(\mathcal{G}) \subset \overline{\operatorname{dec}}\left\{\mathcal{J}_{t}[\overline{\operatorname{co}}(\mathcal{G})]\right\}$. By virtue of ([8], Th. 3.3, Chap. 2) $\overline{\operatorname{dec}}\left\{\mathcal{J}_{t}[\overline{\mathrm{co}}(\mathcal{G})]\right\}$ is a convex subset of the space $\mathbb{L}^{2}\left(\Omega, \mathcal{F}_{t}, \mathbb{R}^{d}\right)$. Then $\overline{\operatorname{co}}\left[\overline{\operatorname{dec}} \mathcal{J}_{t}(\mathcal{G})\right] \subset \overline{\operatorname{dec}}\left\{\mathcal{J}_{t}[\overline{\operatorname{co}}(\mathcal{G})]\right\} . \quad$ Thus, $\overline{\operatorname{dec}}\left\{\mathcal{J}_{t}[\overline{\operatorname{co}}(\mathcal{G})]\right\}=$ $\overline{\mathrm{co}}\left[\overline{\operatorname{dec}} \mathcal{J}_{t}(\mathcal{G})\right]$.

(iv) By separability of the space $(\Omega, \mathcal{F}, P)$ the space $\mathbb{L}^{2}\left(\mathbb{R}^{+} \times \Omega, \Sigma_{\mathbb{F}}, \mathbb{R}^{d \times m}\right)$ is separable. Then $\mathcal{G}$ with its induced topology is a separable subset of $\mathbb{L}^{2}\left(\mathbb{R}^{+} \times \Omega, \Sigma_{\mathbb{F}}, \mathbb{R}^{d \times m}\right)$. Thus there is a sequence $\left(g^{n}\right)_{n=1}^{\infty} \subset \mathcal{G}$ such that $\mathcal{G}=$ $\operatorname{cl}_{\mathbf{I}}\left\{g^{n}: n \geq 1\right\}$, where $\operatorname{cl}_{\mathbf{I}}$ denotes the closure in the induced topology of $\mathcal{G}$. But 
$\operatorname{cl}_{\mathbb{L}}\left\{g^{n}: n \geq 1\right\} \subset \operatorname{cl}_{\mathbb{L}} \mathcal{G}$ and $\operatorname{cl}_{\mathbf{I}}\left\{g^{n}: n \geq 1\right\}=\mathcal{G} \cap \operatorname{cl}_{\mathbb{L}}\left\{g^{n}: n \geq 1\right\}$. Then $\operatorname{cl}_{\mathbb{L}} \mathcal{G}=$ $\operatorname{cl}_{\mathbb{L}}\left[\operatorname{cl}_{\mathbf{I}}\left\{g^{n}: n \geq 1\right\}\right]=\operatorname{cl}_{\mathbb{L}}\left[\mathcal{G} \cap \operatorname{cl}_{\mathbb{L}}\left\{g^{n}: n \geq 1\right\}\right] \subset \operatorname{cl}_{\mathbb{L}} \mathcal{G} \cap \operatorname{cl}_{\mathbb{L}}\left\{g^{n}: n \geq 1\right\}=$ $\operatorname{cl}_{\mathbb{L}}\left\{g^{n}: n \geq 1\right\}$. Hence, and (i) it follows that $\operatorname{cl}_{\mathbb{L}} \mathcal{J}_{t}(\mathcal{G})=\operatorname{cl}_{\mathbb{L}}\left\{\mathcal{J}_{t}\left(g^{n}\right): n \geq 1\right\}$ for every $t \geq 0$.

Lemma 3.2. If $(\Omega, \mathcal{F}, P)$ is separable, $(X, \rho)$ is a metric space and $\Phi: X \ni x \rightarrow$ $\Phi(x) \subset \mathbb{L}^{2}\left(\mathbb{R}^{+} \times \Omega, \Sigma_{\mathbb{F}}, \mathbb{R}^{d \times m}\right)$ is l.s.c. and such that $\Phi(x)$ is a nonempty closed set for every $x \in X$, then there is a sequence $\left(g^{n}\right)_{n=1}^{\infty}$ of continuous functions $g^{n}: X \rightarrow \mathbb{L}^{2}\left(\mathbb{R}^{+} \times \Omega, \Sigma_{\mathbb{F}}, \mathbb{R}^{d \times m}\right)$ such that $g^{n}(x) \in \overline{\operatorname{co}} \Phi(x)$ for $n \geq 1$ and $\overline{\mathrm{CO}} \Phi(x)=\operatorname{cl}_{\mathbb{L}}\left\{g^{n}(x): n \geq 1\right\}$ for every $x \in X$.

Proof. The result follows immediately from ([2], Prop. 4.4, Chap. 1), because the space $\mathbb{L}^{2}\left(\mathbb{R}^{+} \times \Omega, \Sigma_{\mathbb{F}}, \mathbb{R}^{d \times m}\right)$ is separable and a set valued mapping $X \ni$ $x \rightarrow \overline{\mathrm{co}} \Phi(x) \subset \mathbb{L}^{2}\left(\mathbb{R}^{+} \times \Omega, \Sigma_{\mathbb{F}}, \mathbb{R}^{d \times m}\right)$ satisfies the assumptions of ([2], Prop. 4.4, Chap. 1).

Lemma 3.3. If $\mathcal{G}=\left\{g^{n}: n \geq 1\right\} \subset \mathbb{L}^{2}\left(\mathbb{R}^{+} \times \Omega, \Sigma_{\mathbb{F}}, \mathbb{R}^{d \times m}\right)$, then $\overline{\operatorname{dec}} \mathcal{J}_{t}(\mathcal{G})=$ $\operatorname{Lim} \overline{\operatorname{dec}} \mathcal{J}_{t}\left(\mathcal{G}_{p}\right)$, where $\mathcal{G}_{p}=\left\{g^{1}, \ldots, g^{p}\right\}$ for $p \geq 1$.

Proof. Let us observe that $\operatorname{cl}_{\mathbb{L}} \mathcal{G}=\operatorname{Lim} \mathcal{G}_{p}$. Indeed, we have $\mathcal{G}_{p} \subset \mathcal{G}_{p+1}$ for every $p \geq 1$. Therefore, the Kuratowski limit $\operatorname{Lim} \mathcal{G}_{p}$ exists. Furthermore, $\mathcal{G}_{p} \subset$ $\operatorname{cl}_{\mathbb{L}} \mathcal{G}$ for every $p \geq 1$, which implies that $\operatorname{Lim} \mathcal{G}_{p} \subset \operatorname{cl}_{\mathbb{L}} \mathcal{G}$, because $\operatorname{Lim} \mathcal{G}_{p}=$ $\operatorname{cl}_{\mathbb{L}}\left\{\bigcup_{p \geq 1} \mathcal{G}_{p}\right\}$, where the closures are taken with respect to the norm topology of $\mathbb{L}^{2}\left(\mathbb{R}^{+} \times \Omega, \Sigma_{\mathbb{F}}, \mathbb{R}^{d \times m}\right)$. On the other hand, for every $g \in \operatorname{cl}_{\mathbb{L}} \mathcal{G}$ there is a subsequence $\left(g^{n_{k}}\right)_{k=1}^{\infty}$ of $\left(g^{n}\right)_{n=1}^{\infty}$ such that $g^{n_{k}} \rightarrow g$ as $k \rightarrow \infty$. For every $k \geq 1$ there is $p_{k} \geq 1$ such that $g^{n_{k}} \in \mathcal{G}_{p_{k}}$. Then $g \in \overline{\operatorname{Lim}} \mathcal{G}_{p}=\operatorname{Lim} \mathcal{G}_{p}$. Thus $\mathrm{cl}_{\mathbb{L}} \mathcal{G} \subset \operatorname{Lim} \mathcal{G}_{p}$.

In a similar way we obtain $\operatorname{cl}_{\mathbb{L}} \mathcal{J}_{t}(\mathcal{G})=\operatorname{Lim} \mathcal{J}_{t}\left(\mathcal{G}_{p}\right)$, which implies that $\overline{\operatorname{dec}} \mathcal{J}_{t}(\mathcal{G})=\overline{\operatorname{dec}}\left[\operatorname{Lim} \mathcal{J}_{t}\left(\mathcal{G}_{p}\right)\right]$. To the end of the proof, we have to verify that $\overline{\operatorname{dec}}\left[\operatorname{Lim} \mathcal{J}_{t}\left(\mathcal{G}_{p}\right)\right]=\operatorname{Lim} \overline{\operatorname{dec}}\left[\mathcal{J}_{t}\left(\mathcal{G}_{p}\right)\right]$. Let us observe that $\operatorname{Lim} \overline{\operatorname{dec}}\left\{\mathcal{J}_{t}\left(\mathcal{G}_{p}\right)\right\} \subset$ $\overline{\operatorname{dec}}\left\{\operatorname{Lim} \mathcal{J}_{t}\left(\mathcal{G}_{p}\right)\right\}$, because $\overline{\operatorname{dec}}\left\{\mathcal{J}_{t}\left(\mathcal{G}_{p}\right)\right\} \subset \overline{\operatorname{dec}}\left\{\operatorname{Lim} \mathcal{J}_{t}\left(\mathcal{G}_{p}\right)\right\}$ for every $p \geq 1$ and $\overline{\operatorname{dec}}\left\{\operatorname{Lim} \mathcal{J}_{t}\left(\mathcal{G}_{p}\right)\right\}$ is a closed subset of $\mathbb{L}^{2}\left(\Omega, \mathcal{F}, \mathbb{R}^{d}\right)$. For every $a \in \overline{\operatorname{dec}}\left\{\operatorname{Lim} \mathcal{J}_{t}\left(\mathcal{G}_{p}\right)\right\}$ there is a sequence $\left(a_{r}\right)_{r=1}^{\infty}$ of $\left.\operatorname{dec}\left\{\operatorname{Lim} \mathcal{J}_{t}\left(\mathcal{G}_{p}\right)\right\}=\operatorname{dec}\left\{\mathcal{J}_{t}\left[\operatorname{Lim} \mathcal{G}_{p}\right)\right]\right\}$ converging to $a$ in the norm topology of $\mathbb{L}^{2}\left(\Omega, \mathcal{F}, \mathbb{R}^{d}\right)$. For every $r \geq 1$ there are a partition $\left(A_{k}^{r}\right)_{k=1}^{N_{r}} \in \Pi\left(\Omega, \mathcal{F}_{t}\right)$ and a family $\left(u_{k}^{r}\right)_{k=1}^{N_{r}} \subset \mathcal{J}_{t}\left[\operatorname{Lim} \mathcal{G}_{p}\right]$ such that $a_{r}=\sum_{k=1}^{N_{r}} \mathbb{I}_{A_{k}^{r}} u_{k}^{r}$. For every $r \geq 1$ and $k=1, \ldots, N_{r}$ there is a sequence $\left(v_{p}^{k, r}\right)_{p=1}^{\infty}$ such that $v_{p}^{k, r} \in \mathcal{G}_{p}$ for every $p \geq 1$ and $\mathcal{J}_{t}\left(v_{p}^{k, r}\right) \rightarrow u_{k}^{r}$ in the norm topology of $\mathbb{L}^{2}\left(\Omega, \mathcal{F}, \mathbb{R}^{d}\right)$ as $p \rightarrow \infty$. Then, for every $r \geq 1$ we have $\sum_{k=1}^{N_{r}} \mathbb{I}_{A_{k}^{r}} \mathcal{J}_{t}\left(v_{p}^{k, r}\right) \in$ $\operatorname{dec}\left\{\mathcal{J}_{t}\left(\mathcal{G}_{p}\right)\right\}$ for $p \geq 1$ and $\sum_{k=1}^{N_{r}} \mathbb{I}_{A_{k}^{r}} c j_{t}\left(v_{p}^{k, r}\right) \rightarrow \sum_{k=1}^{N_{m}} \mathbb{I}_{A_{k}^{r}} u_{k}^{r}=a_{r}$ as $p \rightarrow \infty$. Therefore, $a_{r} \in \underline{\operatorname{Lim}}\left[\operatorname{dec}\left\{\mathcal{J}_{t}\left(\mathcal{G}_{p}\right)\right\}\right]=\operatorname{Lim}\left[\operatorname{dec}\left\{\mathcal{J}_{t}\left(\mathcal{G}_{p}\right)\right\}\right]$ for every $r \geq 1$, which implies that $a_{r} \in \operatorname{Lim}\left[\overline{\operatorname{dec}}\left\{\mathcal{J}_{t}\left(\mathcal{G}_{p}\right)\right\}\right]$, because $\operatorname{dec} \mathcal{J}_{t}\left(\mathcal{G}_{p}\right) \subset \operatorname{dec} \mathcal{J}_{t}\left(\mathcal{G}_{p+1}\right)$ for every $p \geq 1$. Hence it follows that $a \in \operatorname{Lim}\left[\overline{\operatorname{dec}} \mathcal{J}_{t}\left(\mathcal{G}_{p}\right)\right]$ for every $a \in \overline{\operatorname{dec}}\left\{\operatorname{Lim} \mathcal{J}_{t}\left(\mathcal{G}_{p}\right)\right\}$. 
Thus $\overline{\operatorname{dec}}\left\{\operatorname{Lim} \mathcal{J}_{t}\left(\mathcal{G}_{p}\right)\right\} \subset \operatorname{Lim}\left[\overline{\operatorname{dec}} \mathcal{J}_{t}\left(\mathcal{G}_{p}\right)\right]$, which implies that $\overline{\operatorname{dec}}\left\{\operatorname{Lim} \mathcal{J}_{t}\left(\mathcal{G}_{p}\right)\right\}=$ $\operatorname{Lim} \overline{\operatorname{dec}}\left\{\mathcal{J}_{t}\left(\mathcal{G}_{p}\right)\right\}$.

We present now the basic properties of generalized set-valued stochastic integrals.

Theorem 3.4. For every nonempty set $\mathcal{G} \subset \mathbb{L}^{2}\left(\mathbb{R}^{+} \times \Omega, \Sigma_{\mathbb{F}}, \mathbb{R}^{d \times m}\right)$ we have (i) $\int_{0}^{t} \mathrm{cl}_{\mathbb{L}}(\mathcal{G}) d B_{\tau}=\int_{0}^{t} \mathcal{G} d B_{\tau}$ and (ii) $\int_{0}^{t} \operatorname{co}(\mathcal{G}) d B_{\tau}=\overline{\operatorname{co}} \int_{0}^{t} \mathcal{G} d B_{\tau}$ a.s. for every $t \geq 0$.

Proof. (i) Immediatelly from (i) of Lemma 3.1 we get $\overline{\operatorname{dec}} \mathcal{J}_{t}\left[\operatorname{cl}_{\mathbb{L}}(\mathcal{G})\right]=$ $\left.\overline{\operatorname{dec}}\left[\mathcal{J}_{t}(\mathcal{G})\right]\right\}$ which, by the definition of generalized set-valued stochastic integrals, implies that $S_{t}\left(\int_{0}^{t} \mathrm{cl}_{\mathbb{L}}(\mathcal{G}) d B_{\tau}\right)=S_{t}\left(\int_{0}^{t} \mathcal{G} d B_{\tau}\right)$ for every $t \geq 0$. Then $\int_{0}^{t} \mathrm{cl}_{\mathbb{L}}(\mathcal{G}) d B_{\tau}=\int_{0}^{t} \mathcal{G} d B_{\tau}$ a.s. for every $t \geq 0$.

(ii) Similarly, by (iii) of Lemma 3.1 and the definition of generalized set-valued stochastic integrals, we obtain $S_{t}\left(\int_{0}^{t} \overline{\mathrm{co}}(\mathcal{G}) d B_{\tau}\right)=\overline{\mathrm{co}} S_{t}\left(\int_{0}^{t} \mathcal{G} d B_{\tau}\right)$ for every $t \geq 0$. Immediately from (i) it follows that $\int_{0}^{t} \overline{\mathrm{co}}(\mathcal{G}) d B_{\tau}=\int_{0}^{t} \operatorname{co}(\mathcal{G}) d B_{\tau}$ a.s. for every $t \geq$ 0 . Furthermore, $\overline{\operatorname{co}} S_{t}\left(\int_{0}^{t} \mathcal{G} d B_{\tau}\right)=S_{t}\left(\overline{\operatorname{co}} \int_{0}^{t} \mathcal{G} d B_{\tau}\right)$. Therefore, $S_{t}\left(\int_{0}^{t} \operatorname{co}(\mathcal{G}) d B_{\tau}\right)=$ $S_{t}\left(\overline{\mathrm{co}} \int_{0}^{t} \mathcal{G} d B_{\tau}\right)$ for every $t \geq 0$. Thus $\int_{0}^{t} \operatorname{co}(\mathcal{G}) d B_{\tau}=\overline{\mathrm{co}} \int_{0}^{t} \mathcal{G} d B_{\tau}$ a.s. for every $t \geq 0$.

Theorem 3.5. If $(\Omega, \mathcal{F}, P)$ is separable then for every nonempty set $\mathcal{G} \subset$ $\mathbb{L}^{2}\left(\mathbb{R}^{+} \times \Omega, \Sigma_{\mathbb{F}}, \mathbb{R}^{d \times m}\right)$ there is a sequence $\left(g^{n}\right)_{n=1}^{\infty} \subset \mathcal{G}$ such that $\left(\int_{0}^{t} \mathcal{G} d B_{\tau}\right)(\omega)=$ $\operatorname{cl}\left\{\left(\int_{0}^{t} g_{\tau}^{n} d B_{\tau}\right)(\omega): n \geq 1\right\}$ for every $t \geq 0$ and a.e. $\omega \in \Omega$.

Proof. By (iv) of Lemma 3.1 there is a sequence $\left(g^{n}\right)_{n=1}^{\infty} \subset \mathcal{G}$ such that $\mathrm{cl}_{\mathbb{L}} \mathcal{J}_{t}(\mathcal{G})=\operatorname{cl}_{\mathbb{L}}\left\{\mathcal{J}_{t}\left(g^{n}\right): n \geq 1\right\}$ for every $t \geq 0$, which implies that $\overline{\operatorname{dec}} \mathcal{J}_{t}(\mathcal{G})=$ $\overline{\operatorname{dec}}\left\{\mathcal{J}_{t}\left(g^{n}\right): n \geq 1\right\}$ for every $t \geq 0$. Let $\Gamma_{t}(\omega)=\operatorname{cl}\left\{\left(\int_{0}^{t} g_{\tau}^{n} d B_{\tau}\right)(\omega): n \geq 1\right\}$ for every $t \geq 0$ and $\omega \in \Omega$. We have $S_{t}\left(\Gamma_{t}\right)=\overline{\operatorname{dec}}\left\{\mathcal{J}_{t}\left(g^{n}\right): n \geq 1\right\}$ for every $t \geq 0$, because $\Gamma_{t}$ is an Aumann integrably set-valued random variable. On the other hand, by the definition of generalized set-valued stochastic integrals, we have $S_{t}\left(\int_{0}^{t} \mathcal{G} d B_{\tau}\right)=\overline{\operatorname{dec}} \mathcal{J}_{t}(\mathcal{G})$ for every $t \geq 0$. Then $S_{t}\left(\int_{0}^{t} \mathcal{G} d B_{\tau}\right)=S_{t}\left(\Gamma_{t}\right)$ for every $t \geq 0$, which implies that $\left(\int_{0}^{t} \mathcal{G} d B_{\tau}\right)(\omega)=\operatorname{cl}\left\{\left(\int_{0}^{t} g_{\tau}^{n} d B_{\tau}\right)(\omega): n \geq 1\right\}$ for every $t \geq 0$ and a.e. $\omega \in \Omega$.

Theorem 3.6. If $\mathcal{G}=\left\{g^{n}: n \geq 1\right\} \subset \mathbb{L}^{2}\left(\mathbb{R}^{+} \times \Omega, \Sigma_{\mathbb{F}}, \mathbb{R}^{d \times m}\right)$, then $\int_{0}^{t} \mathcal{G} d B_{\tau}=$ $\operatorname{Lim} \int_{0}^{t} \mathcal{G}_{p} d B_{\tau}$ a.s. for every $t \geq 0$, where $\mathcal{G}_{p}=\left\{g^{1}, \ldots, g^{p}\right\}$ for for $p \geq 1$.

Proof. By virtue of Lemma 3.3 we have $\overline{\operatorname{dec}} \mathcal{J}_{t}(\mathcal{G})=\operatorname{Lim} \overline{\operatorname{dec}} \mathcal{J}_{t}\left(\mathcal{G}_{p}\right)$, for every $t \geq 0$, which implies that $S_{t}\left(\int_{0}^{t} \mathcal{G} d B_{\tau}\right)=\operatorname{Lim} S_{t}\left(\int_{0}^{t} \mathcal{G}_{p} d B_{\tau}\right)$ for $t \geq 0$. We shall show now that $\operatorname{Lim} S_{t}\left(\int_{0}^{t} \mathcal{G}_{p} d B_{\tau}\right)=S_{t}\left(\operatorname{Lim} \int_{0}^{t} \mathcal{G}_{p} d B_{\tau}\right)$ for $t \geq 0$. Indeed, it is clear that $\operatorname{Lim} S_{t}\left(\int_{0}^{t} \mathcal{G}_{p} d B_{\tau}\right) \subset S_{t}\left(\operatorname{Lim} \int_{0}^{t} \mathcal{G}_{p} d B_{\tau}\right)$ for $t \geq 0$. Let $a \in S_{t}\left(\operatorname{Lim} \int_{0}^{t} \mathcal{G}_{p} d B_{\tau}\right)$, i.e., let $a \in \operatorname{Lim} \int_{0}^{t} \mathcal{G}_{p} d B_{\tau}=\underline{\operatorname{Lim}} \int_{0}^{t} \mathcal{G}_{p} d B_{\tau}$ a.s. We have, $d\left(a, \int_{0}^{t} \mathcal{G}_{p} d B_{\tau}\right) \rightarrow 0$ 
a.s. as $p \rightarrow \infty$. Let us observe that the sequence $\left\{d\left(a, \int_{0}^{t} \mathcal{G}_{p} d B_{\tau}\right)\right\}_{p=1}^{\infty}$ is integrably bounded by a function $\varphi:=d\left(a, \int_{0}^{T} g_{t}^{1} d B_{t}\right)$ because $d\left(a, \int_{0}^{t} \mathcal{G}_{p+1} d B_{t}\right) \leq$ $d\left(a, \int_{0}^{t} \mathcal{G}_{p} d B_{\tau}\right)$ for every $p \geq 1$. Therefore, $E\left\{d\left(a, \int_{0}^{t} \mathcal{G}_{p} d B_{\tau}\right)\right\} \rightarrow 0$ as $p \rightarrow \infty$. Hence, by ([1], Th. 2.2) it follows that

$$
\begin{gathered}
d^{2}\left[a, S_{t}\left(\int_{0}^{t} \mathcal{G}_{p} d B_{\tau}\right)\right] \\
=\inf \left\{|a-u|^{2}: u \in S_{t}\left(\int_{0}^{t} \mathcal{G}_{p} d B_{\tau}\right)\right\}=E\left\{d^{2}\left(a, \int_{0}^{t} \mathcal{G}_{p} d B_{\tau}\right)\right\}
\end{gathered}
$$

for every $p \geq 1$, where $\| \cdot \mid$ denotes the norm of the space $\mathbb{L}^{2}\left(\Omega, \mathcal{F}, \mathbb{R}^{d}\right)$. Then $d\left(a, S_{t}\left(\int_{0}^{t} \mathcal{G}_{p} d B_{\tau}\right)\right) \rightarrow 0$ as $p \rightarrow \infty$. Therefore, $a \in \underline{\operatorname{Lim}} S_{t}\left(\int_{0}^{t} \mathcal{G}_{p} d B_{\tau}\right)=$ $\operatorname{Lim} S_{t}\left(\int_{0}^{t} \mathcal{G}_{p} d B_{\tau}\right)$ for every $a \in S_{t}\left(\operatorname{Lim} \int_{0}^{t} \mathcal{G}_{p} B_{\tau}\right)$. Thus $S_{t}\left(\operatorname{Lim} \int_{0}^{t} \mathcal{G}_{p} d B_{\tau}\right) \subset$ $\operatorname{Lim} S_{t}\left(\int_{0}^{t} \mathcal{G}_{p} d B_{\tau}\right)$, which implies that $S_{t}\left(\operatorname{Lim} \int_{0}^{t} \mathcal{G}_{p} d B_{\tau}\right)=\operatorname{Lim} S_{t}\left(\int_{0}^{t} \mathcal{G}_{p} d B_{\tau}\right)$. Now we have $S_{t}\left(\int_{0}^{t} \mathcal{G} d B_{\tau}\right)=S_{t}\left(\operatorname{Lim} \int_{0}^{t} \mathcal{G}^{p} d B_{\tau}\right)$ for every $t \geq 0$, which implies that $\int_{0}^{t} \mathcal{G} d B_{\tau}=\operatorname{Lim} \int_{0}^{t} \mathcal{G}_{p} d B_{\tau}$ a.s. for every $t \geq 0$.

Corollary 3.1. If $(\Omega, \mathcal{F}, P)$ is a separable probability space and $\mathcal{G}$ is a nonempty subset of the space $\mathbb{L}^{2}\left(\mathbb{R}^{+} \times \Omega, \Sigma_{\mathbb{F}}, \mathbb{R}^{d \times m}\right)$ then there exists a sequence $\left(g^{n}\right)_{n=1}^{\infty} \subset$ $\mathcal{G}$ such that $\int_{0}^{t} \mathcal{G} d B_{\tau}=\operatorname{Lim} \int_{0}^{t} \mathcal{G}_{p} d B_{\tau}$ a.s. for every $t \geq 0$, where $\mathcal{G}_{p}=\left\{g^{1}, \ldots, g^{p}\right\}$ for for $p \geq 1$.

Proof. By (iv) of Lemma 3.1 there is a sequence $\left(g^{n}\right)_{n=1}^{\infty} \subset \mathcal{G}$ such that $\operatorname{cl}_{\mathbb{L}} \mathcal{J}_{t}(\mathcal{G})=\operatorname{cl}_{\mathbb{L}}\left\{\mathcal{J}_{t}\left(g^{n}\right): n \geq 1\right\}$ for every $t \geq 0$. Therefore, $\overline{\operatorname{dec}} \mathcal{J}_{t}(\mathcal{G})=$ $\overline{\operatorname{dec}}\left\{\mathcal{J}_{t}\left(g^{n}\right): n \geq 1\right\}$ for every $t \geq 0$, which is equivalent to $S_{t}\left(\int_{0}^{t} \mathcal{G} d B_{\tau}\right)=$ $S_{t}\left(\int_{0}^{t}\left\{g^{n}: n \geq 1\right\} d B_{\tau}\right)$ for every $t \geq 0$. Hence, similarly as in the proof of Theorem 3.6, it follows that $\int_{0}^{t} \mathcal{G} d B_{\tau}=\operatorname{Lim} \int_{0}^{t} \mathcal{G}_{p} d B_{\tau}$ a.s. for every $t \geq 0$.

Remark 3.1. If $\mathcal{G}$ is a nonempty bounded decomposable subset of the space $\mathbb{L}^{2}\left(\mathbb{R}^{+} \times \Omega, \Sigma_{\mathbb{F}}, \mathbb{R}^{d \times m}\right)$ then the last result is true without the assumption that $(\Omega, \mathcal{F}, P)$ is a separable probability space.

Proof. By decomposability of the set $\mathcal{G}$ it follows that there is an $\mathbb{F}$-nonanticipative process $G=\left(G_{t}\right)_{t \geq 0}$ with values in $\mathrm{Cl}\left(\mathbb{R}^{d \times m}\right)$ such that $\mathrm{cl}_{\mathbb{L}} \mathcal{G}=S_{\mathbb{F}}(G)$. By $\Sigma_{\mathbb{F}}$-measurability of $G$ there is a sequence $\left(g^{n}\right)_{n=1}^{\infty}$, of $\Sigma_{\mathbb{F}}$-measurable processes $g^{n}=\left(g_{t}^{n}\right)_{t \geq 0}$, a Castaing representation of $G$, such that $G_{t}(\omega)=\operatorname{cl}\left\{g_{t}^{n}(\omega)\right.$ : $n \geq 1\}$ for every $(t, \omega) \in \mathbb{R}^{+} \times \Omega$. We have $\left(g^{n}\right)_{n=1}^{\infty} \subset \mathbb{L}^{2}\left(\mathbb{R}^{+} \times \Omega, \Sigma_{\mathbb{F}}, \mathbb{R}^{d \times m}\right)$ because $G$ is square integrably bounded. Therefore, $S_{\mathbb{F}}(G)=\overline{\operatorname{dec}}\left\{g^{n}: n \geq 1\right\}$. Thus there is a sequence $\left(g^{n}\right)_{n=1}^{\infty} \subset \mathbb{L}^{2}\left(\mathbb{R}^{+} \times \Omega, \Sigma_{\mathbb{F}}, \mathbb{R}^{d \times m}\right)$ such that $\operatorname{cl}_{\mathbb{L}} \mathcal{G}=$ $\overline{\operatorname{dec}}\left\{g^{n}: n \geq 1\right\}$. Hence, similarly as above (see [9], Th. 3.2) we obtain $\int_{0}^{t} \mathcal{G} d B_{\tau}=$ $\operatorname{Lim} \int_{0}^{t} \operatorname{dec} \mathcal{G}_{p} d B_{\tau}$ a.s. for every $t \geq 0$, where $\mathcal{G}_{p}=\left\{g^{1}, \ldots, g^{p}\right\}$ for for $p \geq 1$. 
Remark 3.2. In a similar way as above we can show that if $\mathcal{G}=\left\{g^{n}: n \geq 1\right\} \subset$ $\mathbb{L}^{2}\left(\mathbb{R}^{+} \times \Omega, \Sigma_{\mathbb{F}}, \mathbb{R}^{d \times m}\right)$, then $\int_{0}^{t} \operatorname{co} \mathcal{G} d B_{\tau}=\operatorname{Lim} \int_{0}^{t} \operatorname{co} \mathcal{G}_{p} d B_{\tau}$ a.s. for every $t \geq 0$, where $\mathcal{G}_{p}=\left\{g^{1}, \ldots, g^{p}\right\}$ for for $p \geq 1$.

We shall show now that for every nonempty set $\mathcal{G} \subset \mathbb{L}^{2}\left(\mathbb{R}^{+} \times \Omega, \Sigma_{\mathbb{F}}, \mathbb{R}^{d}\right)$ such that a generalized set-valued stochastic integral $\int_{0}^{t} \mathcal{G} d B_{\tau}$ of $\mathcal{G}$ with respect to a real $\mathbb{F}$-Brownian motion $B=\left(B_{t}\right)_{t \geq 0}$ is square integrably bounded, we have $\sigma\left(p, \int_{0}^{t} \mathcal{G} d B_{\tau}\right)=\sup \int_{0}^{t} \mathcal{S}(p, \mathcal{G}) d B_{\tau}$ a.s. for every $p \in \mathbb{R}^{d}$ and $t \geq 0 . \quad \sigma(\cdot, A)$ denotes the support function of a set $A \subset \mathbb{R}^{d}, \mathcal{S}(p, \mathcal{G})=\{(p, g): g \in \mathcal{G}\}$ and $(p, g)$ denotes for every $g \in \mathcal{G}$ a real-valued $\mathbb{F}$-nonanticipative stochastic process defined by the inner product $\langle\cdot, \cdot\rangle$ of $\mathbb{R}^{d}$ by setting $(p, g)_{t}(\omega)=\left\langle p, g_{t}(\omega)\right\rangle$ for every $(t, \omega) \in \mathbb{R}^{+} \times \Omega$. Let us note that $\left.\int_{0}^{t} \mathcal{S}(p, \mathcal{G}) d B_{\tau}\right)$ is a closed subset of the real line $\mathbb{R}$.

Theorem 3.7. For every nonempty set $\mathcal{G} \subset \mathbb{L}^{2}\left(\mathbb{R}^{+} \times \Omega, \Sigma_{\mathbb{F}}, \mathbb{R}^{d}\right)$ such that a generalized set-valued stochastic integral $\int_{0}^{t} \mathcal{G} d B_{\tau}$ is square integrably bounded for every $t \geq 0$, we have $\sigma\left(p, \int_{0}^{t} \mathcal{G} d B_{\tau}\right)=\sup \int_{0}^{t} \mathcal{S}(p, \mathcal{G}) d B_{\tau}$ a.s. for every $p \in \mathbb{R}^{d}$ and $t \geq 0$.

Proof. Let $\int_{0}^{t} \mathcal{G} d B_{\tau}$ be square integrably bounded for fixed $t \geq 0$. For every every $p \in \mathbb{R}^{d}$ and $A \in \mathcal{F}_{t}$ we have

$$
\begin{aligned}
& \int_{A} \sigma\left(p, \int_{0}^{t} \mathcal{G} d B_{\tau}\right) d P=\int_{A} \sup \left\{\langle p, x\rangle: x \in \int_{0}^{t} \mathcal{G} d B_{\tau}\right\} d P \\
& =\sup \left\{\int_{A}\langle p, u\rangle d P: u \in S_{t}\left(\int_{0}^{t} \mathcal{G} d B_{\tau}\right)\right\}=\sup \left\{\int_{A}\langle p, u\rangle d P: u \in \operatorname{dec} \mathcal{J}_{t}(\mathcal{G})\right\} \\
& =\sup \left\{\int_{A}\left\langle p, \sum_{k=1}^{N} \mathbb{I}_{A_{k}} \mathcal{J}_{t}\left(g^{k}\right)\right\rangle d P:\left(A_{k}\right)_{k=1}^{N} \in \Pi\left(\Omega, \mathcal{F}_{t}\right),\left(g^{k}\right)_{k=1}^{N} \subset \mathcal{G}\right\} \\
& =\sup \left\{\int_{A}\left[\sum_{k=1}^{N} \mathbb{I}_{A_{k}}\left\langle p, \mathcal{J}_{t}\left(g^{k}\right)\right\rangle\right] d P:\left(A_{k}\right)_{k=1}^{N} \in \Pi\left(\Omega, \mathcal{F}_{t}\right),\left(g^{k}\right)_{k=1}^{N} \subset \mathcal{G}\right\} \\
& =\sup \left\{\int_{A}\left[\sum_{k=1}^{N} \mathbb{I}_{A_{k}} \mathcal{J}_{t}\left(\left\langle p, g^{k}\right\rangle\right)\right] d P:\left(A_{k}\right)_{k=1}^{N} \in \Pi\left(\Omega, \mathcal{F}_{t}\right),\left(g^{k}\right)_{k=1}^{N} \subset \mathcal{G}\right\} \\
& =\sup \left\{\int_{A} u d P: u \in \operatorname{dec} \mathcal{J}_{t}(\mathcal{S}(p, \mathcal{G}))\right\} \\
& =\sup \left\{\int_{A} u d P: u \in S_{t}\left(\int_{0}^{t} \mathcal{S}(p, \mathcal{G}) d B_{\tau}\right)\right\}=\int_{A}\left[\sup \int_{0}^{t} \mathcal{S}(p, \mathcal{G}) d B_{\tau}\right] d P .
\end{aligned}
$$

Therefore, $\sigma\left(p, \int_{0}^{t} \mathcal{G} d B_{\tau}\right)=\sup \int_{0}^{t} \mathcal{S}(p, \mathcal{G}) d B_{\tau}$ a.s. for every $p \in \mathbb{R}^{d}$ and fixed $t \geq 0$. 


\section{INTEGRABLE BOUNDEDNESS OF GENERALIZED SET-VALUED INTEGRALS}

We present now some results dealing with integrable boundedness of generalized set-valued stochastic integrals. We begin with the following lemma.

Lemma 4.1. For every $m$-dimensional $\mathbb{F}$-Brownian motion $B=\left(B_{t}\right)_{t \geq 0}$ defined on a complete filtered probability space $\mathcal{P}_{\mathbb{F}}$, and every set $\mathcal{G}_{p}=\left\{g^{i}: 1 \leq i \leq p\right\} \subset$ $\mathbb{L}^{2}\left(\mathbb{R}^{+} \times \Omega, \Sigma_{\mathbb{F}}, \mathbb{R}^{d \times m}\right)$ one has $E\left\|\int_{0}^{t} \mathcal{G}_{p} d B_{\tau}\right\|^{2} \leq p \cdot E \int_{0}^{t} \max _{1 \leq i \leq p}\left|g_{\tau}^{i}\right|^{2} d \tau<\infty$ for every $t \geq 0$.

Proof. By virtue of ([1], Th. 2.2) we get

$$
\begin{aligned}
& E\left\|\int_{0}^{t} \mathcal{G}_{p} d B_{\tau}\right\|^{2}=\sup \left\{E|u|^{2}: u \in S_{t}\left(\int_{0}^{t} \mathcal{G}_{p} d B_{\tau}\right)\right\} \\
& =\sup \left\{E|u|^{2}: u \in \operatorname{dec}\left\{\mathcal{J}_{t}\left(g^{1}\right), \ldots, \mathcal{J}_{t}\left(g^{p}\right)\right\}\right\} \\
& =\sup \left\{E\left[\sum_{j=1}^{p} \mathbb{I}_{A_{j}}\left|\mathcal{J}_{t}\left(g^{j}\right)\right|^{2}\right]:\left(A_{j}\right)_{j=1}^{p} \in \Pi\left(\Omega, \mathcal{F}_{t}\right)\right\} \\
& \leq \sum_{j=1}^{p} E \int_{0}^{t}\left|g_{\tau}^{j}\right|^{2} d \tau \leq p \cdot E \int_{0}^{t} \max _{1 \leq i \leq p}\left|g_{\tau}^{i}\right|^{2} d \tau<\infty
\end{aligned}
$$

We shall prove now the following theorem dealing with integrable boundedness of generalized set-valued stochastic integrals.

Theorem 4.2. Let $\mathcal{G}$ be a nonempty subset of $\mathbb{L}^{2}\left(\mathbb{R}^{+} \times \Omega, \Sigma_{\mathbb{F}}, \mathbb{R}^{d \times m}\right)$. If $\int_{0}^{t} \mathcal{G} d B_{\tau}$ is square integrably bounded for every $t \geq 0$ then $\mathbb{1}_{[0, t]} \mathcal{G}$ is a bounded subset of $\mathbb{L}^{2}\left(\mathbb{R}^{+} \times \Omega, \Sigma_{\mathbb{F}}, \mathbb{R}^{d \times m}\right)$ every $t \geq 0$.

Proof. Let $\int_{0}^{t} \mathcal{G} d B_{\tau}$ be square integrably bounded for fixed $t \geq 0$. Then $S_{t}\left(\int_{0}^{t} \mathcal{G} d B_{\tau}\right)$ is a boundedd subset of $\mathbb{L}^{2}\left(\Omega, \mathcal{F}_{t}, \mathbb{R}^{d}\right)$. Thus there exists $M_{t}>0$ such that $E|u|^{2} \leq M_{t}$ for every $u \in S_{t}\left(\int_{0}^{t} \mathcal{G} d B_{\tau}\right)$. But $S_{t}\left(\int_{0}^{t} \mathcal{G} d B_{\tau}\right)=\overline{\operatorname{dec}} \mathcal{J}_{t}(\mathcal{G})$. Therefore, $\mathcal{J}_{t}(g) \in S_{t}\left(\int_{0}^{t} \mathcal{G} d B_{\tau}\right)$ for every $g \in \mathcal{G}$, which implies that $\left\|\mathbb{1}_{[0, t]} g\right\|^{2}=$ $E \int_{0}^{t}\left|g_{\tau}\right|^{2} d \tau=E\left|\mathcal{J}_{t}(g)\right|^{2} \leq M_{t}$ for every $g \in \mathcal{G}$. Thus $\mathbb{1}_{[0, t]} \mathcal{G}$ is a bounded subset of $\mathbb{L}^{2}\left(\mathbb{R}^{+} \times \Omega, \Sigma_{\mathbb{F}}, \mathbb{R}^{d \times m}\right)$.

Let us observe that boundedness of a set $\mathbb{1}_{[0, t]} \mathcal{G}$ is not sufficient for square integrable boundedness of a generalized set-valued integral $\int_{0}^{t} \mathcal{G} d B_{\tau}$. Indeed, by virtue of results of [11] there exists an integrably bounded set-valued $\mathbb{F}$-nonanticipative process $G=\left(G_{t}\right)_{t \geq 0}$ such that $S_{\mathbb{F}}(G)$ is a bounded subset of $\mathbb{L}^{2}\left(\mathbb{R}^{+} \times \Omega, \Sigma_{\mathbb{F}}\right.$, $\left.\mathbb{R}^{d \times m}\right)$ and $E\left\|\int_{0}^{t} G_{\tau} d B_{\tau}\right\|^{2}=\infty$. Taking $\mathcal{G}=S_{\mathbb{F}}(G)$ we obtain for every fixed 
$t \geq 0$ a bounded subset $\mathbb{1}_{[0, t]} \mathcal{G}$ of $\mathbb{L}^{2}\left(\mathbb{R}^{+} \times \Omega, \Sigma_{\mathbb{F}}, \mathbb{R}^{d \times m}\right)$ such that $E\left\|\int_{0}^{t} \mathcal{G} d B_{\tau}\right\|^{2}$ $=\infty$, because $\int_{0}^{t} \mathcal{G} d B_{\tau}=\int_{0}^{t} G_{\tau} d B_{\tau}$.

We shall present now the following sufficient condition for square integrable boundedness of generalized set-valued stochastic integrals.

Theorem 4.3. Let $\mathcal{G} \subset \mathbb{L}^{2}\left(\mathbb{R}^{+} \times \Omega, \Sigma_{\mathbb{F}}, \mathbb{R}^{d \times m}\right)$ be a nonempty set and $\left(g^{n}\right)_{n=1}^{\infty} \subset$ $\mathcal{G}$ a sequence such that $\operatorname{cl}_{\mathbb{L}} \mathcal{G}=\operatorname{cl}_{\mathbb{L}}\left\{g^{n}: n \geq 1\right\}$ and $\sum_{n=1}^{\infty}\left\|g^{n}\right\|^{2}<\infty$, where $\|\cdot\|$ is the norm of $\mathbb{L}^{2}\left(\mathbb{R}^{+} \times \Omega, \Sigma_{\mathbb{F}}, \mathbb{R}^{d \times m}\right)$. Then a generalized set-valued stochastic integral $\int_{0}^{t} \mathcal{G} d B_{\tau}$ is square integrably bounded for every $t \geq 0$.

Proof. Let $\sum_{n=1}^{\infty}\left\|g^{n}\right\|^{2}<\infty$. Similarly as in the proof of Lemma 4.1, for every $p \geq 1$ we obtain

$$
E\left\|\int_{0}^{t} \mathcal{G}_{p} d B_{\tau}\right\|^{2} \leq \sum_{j=1}^{p} E \int_{0}^{t}\left|g_{\tau}^{j}\right|^{2} d \tau \leq \sum_{n=1}^{\infty}\left\|g^{n}\right\|^{2}<\infty,
$$

where $\mathcal{G}_{p}=\left\{g^{1}, \ldots, g^{p}\right\}$. By Theorem 3.6 we have $\int_{0}^{t} \mathcal{G} d B_{\tau}=\operatorname{Lim} \int_{0}^{t} \mathcal{G}_{p} d B_{\tau}$ a.s. for every $t \geq 0$. Therefore, for every $t \geq 0$ we get

$$
\begin{aligned}
& E\left\|\int_{0}^{t} \mathcal{G} d B_{\tau}\right\|^{2}=\sup \left\{E|u|^{2}: u \in S_{t}\left(\operatorname{Lim} \int_{0}^{t} \mathcal{G}_{p} d B_{\tau}\right)\right\} \\
& =\sup \left\{E|u|^{2}: u \in \operatorname{Lim} S_{t}\left(\int_{0}^{t} \mathcal{G}_{p} d B_{\tau}\right)\right\} \\
& =\sup \left\{E|u|^{2}: u \in \bigcup_{p=1}^{\infty}\left[S_{t}\left(\int_{0}^{t} \mathcal{G}_{p} d B_{\tau}\right)\right]\right\} \leq \sup _{p \geq 1}\left\{E|u|^{2}: u \in S_{t}\left(\int_{0}^{t} \mathcal{G}_{p} d B_{\tau}\right)\right\} .
\end{aligned}
$$

Hence, by ([1], Th. 2.2) it follows that $S_{t}\left(\int_{0}^{t} \mathcal{G} d B_{\tau}\right)$ is a bounded subset in the space $\mathbb{L}^{2}\left(\Omega, \mathcal{F}_{t}, \mathbb{R}^{d}\right)$. Thus a set-valued integral $\int_{0}^{t} \mathcal{G}_{\tau} d B_{\tau}$ is square integrably bounded for every $t \geq 0$.

Corollary 4.1. For every set $\mathcal{G}_{p}=\left\{g^{i}: 1 \leq i \leq p\right\} \subset \mathbb{L}^{2}\left(\mathbb{R}^{+} \times \Omega, \Sigma_{\mathbb{F}}, \mathbb{R}^{d \times m}\right)$ a generalized set-valued stochastic integral $\int_{0}^{t} \mathcal{G}_{p} d B_{\tau}$ is square integrably bounded for every $t \geq 0$.

Remark 4.1. Immediately from (iii) of Lemma 3.1 and (ii) of Theorem 3.4 it follows that for every nonempty set $\mathcal{G} \subset \mathbb{L}^{2}\left(\mathbb{R}^{+} \times \Omega, \Sigma_{\mathbb{F}}, \mathbb{R}^{d \times m}\right)$ a generalized set-valued stochastic integral $\int_{0}^{t} \mathcal{G} d B_{\tau}$ is square integrably bounded if and only if $\int_{0}^{t} \operatorname{co} \mathcal{G} d B_{\tau}$ is square integrably bounded.

We shall prove now some results dealing with estimations of the Hausdorff distance between generalized set-valued stochastic integrals. 
Theorem 4.3. For every m-dimensional $\mathbb{F}$-Brownian motion $B=\left(B_{t}\right)_{t \geq 0}$ defined on a complete filtered probability space $\mathcal{P}_{\mathbb{F}}$, and every sets $\left\{f^{i}: 1 \leq i \leq p\right\}$, $\left\{g^{i}: 1 \leq i \leq p\right\} \subset \mathbb{L}^{2}\left(\mathbb{R}^{+} \times \Omega, \Sigma_{\mathbb{F}}, \mathbb{R}^{d \times m}\right)$ one has

$$
\begin{aligned}
& E h^{2}\left(\int_{0}^{t}\left\{f^{i}: 1 \leq i \leq p\right\} d B_{\tau}, \int_{0}^{t}\left\{g^{i}: 1 \leq i \leq p\right\} d B_{\tau}\right) \\
& \leq p \cdot H^{2}\left(\left\{f^{1}, \ldots, f^{p}\right\},\left\{g^{1}, \ldots, g^{p}\right\}\right)
\end{aligned}
$$

where $H$ is the Hausdorff metric in $\mathbb{L}^{2}\left(\mathbb{R}^{+} \times \Omega, \Sigma_{\mathbb{F}}, \mathbb{R}^{d \times m}\right)$.

Proof. Let $p \geq 1$ be fixed. By virtue of ([1], Th. 2.2) we have

$$
\begin{aligned}
E \bar{h}^{2}\left(\int_{0}^{t}\left\{f^{i}: 1 \leq i \leq p\right\} d B_{\tau}, \int_{0}^{t}\left\{g^{i}: 1 \leq i \leq p\right\} d B_{\tau}\right) \\
=\sup \left\{\inf \left\{E|u-v|^{2}: v \in S_{t}\left(\int_{0}^{t}\left\{g^{i}: 1 \leq i \leq p\right\} d B_{\tau}\right)\right\}:\right. \\
\left.\quad u \in S_{t}\left(\int_{0}^{t}\left\{f^{i}: 1 \leq i \leq p\right\} d B_{\tau}\right)\right\} \\
=\sup \left\{\inf \left\{E|u-v|^{2}: v \in \operatorname{dec}\left\{\mathcal{J}_{t}\left(g^{i}\right): 1 \leq i \leq p\right\}\right\}: u \in \operatorname{dec}\left\{\mathcal{J}_{t}\left(f^{i}\right): 1 \leq i \leq p\right\}\right\} .
\end{aligned}
$$

For every $u \in \operatorname{dec}\left\{\mathcal{J}_{t}\left(f^{i}\right): 1 \leq i \leq p\right\}$ one has

$$
\inf \left\{E|u-v|^{2}: v \in \operatorname{dec}\left\{\mathcal{J}_{t}\left(g^{i}\right): 1 \leq i \leq p\right\}\right\} \leq \min \left\{E\left|u-\mathcal{J}_{t}\left(g^{i}\right)\right|^{2}: 1 \leq i \leq p\right\} .
$$

On the other hand, for every $u \in \operatorname{dec}\left\{\mathcal{J}_{t}\left(f^{i}\right): 1 \leq i \leq p\right\}$ there is $\left(A_{j}\right)_{j=1}^{p} \in$ $\Pi\left(\Omega, \mathcal{F}_{t}\right)$ such that $u=\sum_{j=1}^{p} \mathbb{I}_{A_{j}} \mathcal{J}_{t}\left(f^{j}\right)$. Then

$$
\begin{aligned}
& E \bar{h}^{2}\left(\int_{0}^{t}\left\{f^{i}: 1 \leq i \leq p\right\} d B_{\tau}, \int_{0}^{t}\left\{g^{i}: 1 \leq i \leq p\right\} d B_{\tau}\right) \\
& \leq \sup \left\{\min _{1 \leq i \leq p} E\left[\sum_{j=1}^{p} \mathbb{I}_{A_{j}}\left|\mathcal{J}_{t}\left(f^{j}\right)-\mathcal{J}_{t}\left(g^{i}\right)\right|^{2}\right]:\left(A_{j}\right)_{j=1}^{p} \in \Pi\left(\Omega, \mathcal{F}_{t}\right)\right\} \\
& \leq p \cdot \max _{1 \leq j \leq p}\left\{\min _{1 \leq i \leq p} E\left|\mathcal{J}_{t}\left(f^{j}\right)-\mathcal{J}_{t}\left(g^{i}\right)\right|^{2}\right\} \leq p \cdot H^{2}\left(\left\{f^{1}, \ldots, f^{p}\right\},\left\{g^{1}, \ldots, g^{p}\right\}\right) .
\end{aligned}
$$

In a similar way we also get $E \bar{h}^{2}\left(\int_{0}^{t}\left\{g^{i}: 1 \leq i \leq p\right\} d B_{\tau}, \int_{0}^{t}\left\{f^{i}: 1 \leq i \leq p\right\} d B_{\tau}\right) \leq$ $p \cdot H^{2}\left(\left\{f^{1}, \ldots, f^{p}\right\},\left\{g^{1}, \ldots, g^{p}\right\}\right)$. Then (4.1) is satisfied. 
Corollary 4.2. If the assumptions of Theorem 4.3 are satified then

$$
\begin{aligned}
& \text { (4.2) } \quad E h^{2}\left(\int_{0}^{t} \operatorname{co}\left\{f^{i}: 1 \leq i \leq p\right\} d B_{\tau}, \int_{0}^{t} \operatorname{co}\left\{g^{i}: 1 \leq i \leq p\right\} d B_{\tau}\right) \\
& \leq E h^{2}\left(\int_{0}^{t}\left\{f^{i}: 1 \leq i \leq p\right\} d B_{\tau}, \int_{0}^{t}\left\{g^{i}: 1 \leq i \leq p\right\} d B_{\tau}\right) \leq p \cdot E \int_{0}^{t} \max _{1 \leq j \leq p}\left|f_{\tau}^{j}-g_{\tau}^{j}\right|^{2} d \tau .
\end{aligned}
$$

Remark 4.2. In a similar way we can verify that for every sequences $\left(f^{n}\right)_{n=1}^{\infty}$, $\left(g^{n}\right)_{n=1}^{\infty} \subset \mathbb{L}^{2}\left(\mathbb{R}^{+} \times \Omega, \Sigma_{\mathbb{F}}, \mathbb{R}^{d \times m}\right)$ such that $\sum_{n=1}^{\infty}\left\|f^{n}\right\|^{2}<\infty$, and $\sum_{n=1}^{\infty}\left\|g^{n}\right\|^{2}$ $<\infty$, one has

$$
E h^{2}\left(\int_{0}^{t} \mathcal{F} d B_{\tau}, \int_{0}^{t} \mathcal{G} d B_{\tau}\right) \leq E \int_{0}^{t} \sup _{j \geq 1}\left|f_{\tau}^{j}-g_{\tau}^{j}\right|^{2} d \tau
$$

where $\mathcal{F}=\operatorname{co}\left\{f^{n}: n \geq 1\right\}$ and $\mathcal{G}=\operatorname{co}\left\{g^{n}: n \geq 1\right\}$.

\section{Generalized indefinite Set-Valued stochastic integrals}

Throughout this section we shall consider a nonempty set $\mathcal{G}^{p}=\overline{\mathrm{co}}\left\{g^{1}, \ldots, g^{p}\right\}$, for a given finite set $\left\{g^{1}, \ldots, g^{p}\right\} \subset \mathbb{L}^{2}\left(\mathbb{R}^{+} \times \Omega, \Sigma_{\mathbb{F}}, \mathbb{R}^{d \times m}\right)$. Given an $m$-dimensional $\mathbb{F}$-Brownian motion $B=\left(B_{t}\right)_{t \geq 0}$ defined on $\mathcal{P}_{\mathbb{F}}$, the set-valued $\mathbb{F}$-adapted stochastic process $\left(\int_{0}^{t} \mathcal{G}^{p} d B_{\tau}\right)_{t \geq 0}$ is called the generalized indefinite set-valued stochastic integral of $\mathcal{G}_{p}$. Immediately from Lemma 4.1 and the properties of the Hausdorff metric $h$ we have $E\left\|\int_{0}^{t} \mathcal{G}^{p} d B_{\tau}\right\|^{2} \leq p \cdot E \int_{0}^{t} \max _{1 \leq i \leq p}\left|g_{\tau}^{i}\right|^{2} d \tau$. We shall prove that the generalized indefinite set-valued stochastic integral $\left(\int_{0}^{t} \mathcal{G}^{p} d B_{\tau}\right)_{t \geq 0}$ is a set-valued submartingale, i.e., such that $\int_{0}^{s} \mathcal{G}^{p} d B_{\tau} \subset E\left[\int_{0}^{t} \mathcal{G}^{p} d B_{\tau} \mid \mathcal{F}_{s}\right]$ a.s. for every $0 \leq s<t<\infty$. To do that let us observe (see [7], Th. 3.2) that, by square integrable boundedness of a set-valued generalized set-valued stochastic integral $\int_{0}^{t} \mathcal{G}^{p} d B_{\tau}$, we have $\int_{0}^{t} \mathcal{G}^{p} d B_{\tau}=\int_{0}^{s} \mathcal{G}^{p} d B_{\tau}+\int_{s}^{t} \mathcal{G}^{p} d B_{\tau}$ a.s. for every $0 \leq s<t \leq<\infty$.

Lemma 5.1. Let $B=\left(B_{t}\right)_{t \geq 0}$ be an m-dimensional $\mathbb{F}$-Brownian motion defined on $\mathcal{P}_{\mathbb{F}}$ and $\mathcal{G}^{p} \subset \mathbb{L}^{2}\left(\mathbb{R}^{+} \times \Omega, \Sigma_{\mathbb{F}}, \mathbb{R}^{d \times m}\right)$ be such as above. For every $0 \leq s<$ $t<\infty$ one has $\int_{0}^{s} \mathcal{G}^{p} d B_{\tau}=E\left[\mathcal{J}_{t}\left(\mathcal{G}^{p}\right) \mid \mathcal{F}_{s}\right]$ a.s. for every $p \geq 1$.

Proof. By the definitions of generalized set-valued integrals and the generalized set-valued conditional expectations we get

$$
S_{\mathcal{F}_{s}}\left(\int_{0}^{s} \mathcal{G}^{p} d B_{\tau}\right)=\overline{\operatorname{dec}}_{\mathcal{F}_{s}}\left\{\mathcal{J}_{s}(g): g \in \mathcal{G}^{p}\right\}
$$




$$
\begin{aligned}
& =\overline{\operatorname{dec}}_{\mathcal{F}_{s}}\left\{E\left[\mathcal{J}_{t}(g) \mid \mathcal{F}_{s}\right]: g \in \mathcal{G}^{p}\right\}=\overline{\operatorname{dec}}_{\mathcal{F}_{s}}\left\{E\left[u \mid \mathcal{F}_{s}\right]: u \in \mathcal{J}_{t}\left(\mathcal{G}^{p}\right)\right\} \\
& =S_{\mathcal{F}_{s}}\left(E\left[\mathcal{J}_{t}\left(\mathcal{G}^{p}\right) \mid \mathcal{F}_{s}\right]\right) .
\end{aligned}
$$

Then $\left.\int_{0}^{s} \mathcal{G}^{p} d B_{\tau}=E\left[\mathcal{J}_{t}\left(\mathcal{G}^{p}\right) \mid \mathcal{F}_{s}\right]\right]$ a.s. for every $0 \leq s<t<\infty$.

Corollary 5.1. If the assamptions of Lemma 5.1 are satisfied then a set-valued stochastic process $\left(\int_{0}^{t} \mathcal{G}^{p} d B_{\tau}\right)_{t \geq 0}$ is a set-valued submartingale for every $p \geq 1$.

Proof. The result follows immediately from Lemma 5.1, an inclusion $E\left[\mathcal{J}_{t}\left(\mathcal{G}^{p}\right) \mid \mathcal{F}_{s}\right] \subset E\left[\overline{\operatorname{dec}}_{\mathcal{F}_{t}}\left\{\mathcal{J}_{t}\left(\mathcal{G}^{p}\right)\right\} \mid \mathcal{F}_{s}\right]$ for $0 \leq s<t<\infty$ and the equality $S_{t}\left(\int_{0}^{t} \mathcal{G}^{p} d B_{\tau}\right)=\overline{\operatorname{dec}}_{\mathcal{F}_{t}}\left\{\mathcal{J}_{t}\left(\mathcal{G}^{p}\right)\right\}$. Indeed, we have

$$
E\left[\overline{\operatorname{dec}}_{\mathcal{F}_{t}}\left\{\mathcal{J}_{t}\left(\mathcal{G}^{p}\right)\right\} \mid \mathcal{F}_{s}\right]=E\left[S_{t}\left(\int_{0}^{t} \mathcal{G}^{p} d B_{\tau}\right) \mid \mathcal{F}_{s}\right]=E\left[\int_{0}^{t} \mathcal{G}^{p} d B_{\tau} \mid \mathcal{F}_{s}\right]
$$

Then $E\left[\mathcal{J}_{t}\left(\mathcal{G}^{p}\right) \mid \mathcal{F}_{s}\right] \subset E\left[\int_{0}^{t} \mathcal{G}^{p} d B_{\tau} \mid \mathcal{F}_{s}\right]$, for $0 \leq s<t<\infty$, which implies that

$$
S_{\mathcal{F}_{s}}\left(\int_{0}^{s} \mathcal{G}^{p} d B_{\tau}\right)=S_{\mathcal{F}_{s}}\left(E\left[\mathcal{J}_{t}\left(\mathcal{G}^{p}\right) \mid \mathcal{F}_{s}\right]\right) \subset S_{\mathcal{F}_{s}}\left(E\left[\int_{0}^{t} \mathcal{G}^{p} d B_{\tau} \mid \mathcal{F}_{s}\right]\right)
$$

for every $0 \leq s<t \leq T$. Thus $\int_{0}^{s} \mathcal{G}^{p} d B_{\tau} \subset E\left[\int_{0}^{t} \mathcal{G}^{p} d B_{\tau} \mid \mathcal{F}_{s}\right]$ a.s. for every $0 \leq s<t \leq T$.

Lemma 5.2. Let $B=\left(B_{t}\right)_{t \geq 0}$ be an m-dimensional $\mathbb{F}$-Brownian motion defined on $\mathcal{P}_{\mathbb{F}}$, and $\mathcal{G}^{p} \subset \mathbb{L}^{2}\left(\mathbb{R}^{+} \times \Omega, \Sigma_{\mathbb{F}}, \mathbb{R}^{d \times m}\right)$ be such that as above. A real-valued process $\left(\left\|\int_{0}^{t} \mathcal{G}^{p} d B_{\tau}\right\|\right)_{t \geq 0}$ is a positive submartingale.

Proof. By Corollary 5.1 the set-valued process $\left(\int_{0}^{t} \mathcal{G}^{p} d B_{\tau}\right)_{t \geq 0}$ is a set-valued submartingale. Therefore, $\left\|\int_{0}^{s} \mathcal{G}^{p} d B_{\tau}\right\| \leq\left\|E\left[\int_{0}^{t} \mathcal{G}^{p} d B_{\tau} \mid \mathcal{F}_{s}\right]\right\|$ a.s. for every $0 \leq s<$ $t<\infty$. By ([1], Th. 5.2) it follows that $\left\|E\left[\int_{0}^{t} \mathcal{G}^{p} d B_{\tau} \mid \mathcal{F}_{s}\right]\right\| \leq E\left[\left\|\int_{0}^{t} \mathcal{G}^{p} d B_{\tau}\right\| \mid \mathcal{F}_{s}\right]$ a.s. for every $0 \leq s<t<\infty$. Then, $\left\|\int_{0}^{s} \mathcal{G}^{p} d B_{\tau}\right\| \leq E\left[\left\|\int_{0}^{t} \mathcal{G}^{p} d B_{\tau}\right\| \mathcal{F}_{s}\right]$ a.s. for every $0 \leq s<t<\infty$.

Immediately from the definition of a generalized set-valued integrals it follows that they are $\mathbf{F}$-adapted. We shall prove now that by the assumptions of Lemma 5.2 , they are also continuous. We begin with the following lemma.

Lemma 5.3. Let $B=\left(B_{t}\right)_{t \geq 0}$ be an $m$-dimensional $\mathbb{F}$-Brownian motion, $T>0$ and $\mathcal{G}^{p} \subset \mathbb{L}^{2}\left(\mathbb{R}^{+} \times \Omega, \Sigma_{\mathbb{F}}, \mathbb{R}^{d \times m}\right)$ be such as above. For every $\lambda>0$ one has 


$$
P\left(\left\{\sup _{0 \leq t \leq T}\left\|\int_{0}^{t} \mathcal{G}^{p} d B_{\tau}\right\| \geq \lambda\right\}\right) \leq p / \lambda^{2} E \int_{0}^{T} \max _{1 \leq k \leq p}\left|g_{\tau}^{k}\right|^{2} \tau .
$$

Proof. It was proved in Lemma 5.2, that a real-valued stochastic process $\left(\left\|\int_{0}^{t} \mathcal{G} d B_{\tau}\right\|\right)_{t \geq 0}$ is a positive submartingale. Now, the result follows immediately from Chebyshev's and Doob's inequalities, and Lemma 4.1. Indeed, one has

$$
\begin{aligned}
& P\left(\left\{\sup _{0 \leq t \leq T}\left\|\int_{0}^{t} \mathcal{G}^{p} d B_{\tau}\right\| \geq \lambda\right\}\right) \leq 1 / \lambda^{2} E\left[\sup _{0 \leq t \leq T}\left\|\int_{0}^{t} \mathcal{G}^{p} d B_{\tau}\right\|^{2}\right] \\
& \leq 1 / \lambda^{2} \sup _{0 \leq t \leq T} E\left[\left\|\int_{0}^{t} \mathcal{G}^{p} d B_{\tau}\right\|^{2}\right] \leq p / \lambda^{2} E \int_{0}^{T} \max _{1 \leq k \leq p}\left|g_{\tau}^{k}\right|^{2} d \tau .
\end{aligned}
$$

We shall prove now that a set-valued stochastic process $\left(\int_{0}^{t} \mathcal{G}^{p} d B_{\tau}\right)_{t \geq 0}$ is continuous.

Theorem 5.4. Let $B=\left(B_{t}\right)_{t \geq 0}$ be an m-dimensional $\mathbb{F}$-Brownian motion defined on $\mathcal{P}_{\mathbb{F}}$. For every finite set $\left\{g^{1}, \ldots, g^{p}\right\} \subset \mathbb{L}^{2}\left(\mathbb{R}^{+} \times \Omega, \Sigma_{\mathbb{F}}, \mathbb{R}^{d \times m}\right)$ a setvalued stochastic process $\left(\int_{0}^{t} \mathcal{G}^{p} d B_{\tau}\right)_{t \geq 0}$ with $\mathcal{G}^{p}=\overline{\mathrm{co}}\left\{g^{1}, \ldots, g^{p}\right\}$ is continuous.

Proof. Let $\left\{g^{1}, \ldots, g^{p}\right\} \subset \mathbb{L}^{2}\left(\mathbb{R}^{+} \times \Omega, \Sigma_{\mathbb{F}}, \mathbb{R}^{d \times m}\right)$ be given. By the additivity property of generalized set-valued stochastic integrals and Lemma 5.3, for every $t_{0} \geq 0, \delta>0$ and $n \geq 1$ we obtain

$$
\begin{aligned}
& P\left[\left\{\sup _{t_{0} \leq t \leq t_{0}+\delta} h\left(\int_{0}^{t} \mathcal{G}^{p} d B_{\tau}, \int_{0}^{t_{0}} \mathcal{G}^{p} d B_{\tau}\right)>1 / 2^{n}\right\}\right] \\
& =P\left[\left\{\sup _{t_{0} \leq t \leq t_{0}+\delta} h\left(\int_{0}^{t_{0}} \mathcal{G}^{p} d B_{\tau}+\int_{t_{0}}^{t} \mathcal{G}^{p} d B_{\tau}, \int_{0}^{t_{0}} \mathcal{G}^{p} d B_{\tau}\right)>1 / 2^{n}\right\}\right] \\
& =P\left[\left\{\sup _{t_{0} \leq t \leq t_{0}+\delta} h\left(\int_{t_{0}}^{t} \mathcal{G}^{p} d B_{\tau},\{0\}\right)>1 / 2^{n}\right\}\right] \\
& =P\left[\left\{\sup _{t_{0} \leq t \leq t_{0}+\delta}\left\|\int_{t_{0}}^{t} \mathcal{G}^{p} d B_{\tau}\right\|>1 / 2^{n}\right\}\right] \leq 2^{2 n} p \cdot E \int_{t_{0}}^{t_{0}+\delta} \max _{1 \leq k \leq p}\left|g_{\tau}^{k}\right|^{2} d \tau .
\end{aligned}
$$

For every $n \geq 1$ there is $\delta_{n}>0$ such that $E \int_{t_{0}}^{t_{0}+\delta_{n}} \max _{1 \leq k \leq p}\left|g_{\tau}^{k}\right|^{2} d \tau \leq 1 /\left(p \cdot 2^{3 n}\right)$. Therefore, for every $n \geq 1$ one has

$$
P\left[\left\{\sup _{t_{0} \leq t \leq t_{0}+\delta_{n}} h\left(\int_{0}^{t} \mathcal{G}^{p} d B_{\tau}, \int_{0}^{t_{0}} \mathcal{G}^{p} d B_{\tau}\right)>1 / 2^{n}\right\}\right] \leq 1 / 2^{n}
$$


which implies that $\sum_{n=1}^{\infty} P\left(\Lambda_{n}\right)<\infty$, where

$$
\Lambda_{n}=\left\{\omega \in \Omega: \sup _{t_{0} \leq t \leq t_{0}+\delta_{n}} h\left[\left(\int_{0}^{t} \mathcal{G}^{p} d B_{\tau}\right)(\omega),\left(\int_{0}^{t_{0}} \mathcal{G}^{p} d B_{\tau}\right)(\omega)\right]>1 / 2^{n}\right\} .
$$

Therefore, by the Borel-Cantelli lemma we have $P\left(\bigcap_{n \geq 1} \bigcup_{k \geq n} \Lambda_{k}\right)=0$, which is equivalent to $P\left(\bigcup_{n \geq 1} \bigcap_{k \geq n} \Lambda_{k}^{\sim}\right)=1$, where $\Lambda_{k}^{\sim}=\Omega \backslash \Lambda_{k}$. Hence it follows that for every $\omega \in \bigcup_{n \geq 1} \bigcap_{k \geq n} \Lambda_{k}^{\tilde{k}}$ there is $n(\omega) \geq 1$ such that for every $k \geq n(\omega)$ one has $\sup _{t_{0} \leq t \leq t_{0}+\delta_{k}} h\left[\left(\int_{0}^{t} \mathcal{G} d B_{\tau}\right)(\omega),\left(\int_{0}^{t_{0}} \mathcal{G} d B_{\tau}\right)(\omega)\right] \leq 1 / 2^{k}$, that can be written in the form $\left.\sup _{t_{0} \leq t \leq t_{0}+\delta_{k}} \| \int_{t_{0}}^{t} \mathcal{G} d B_{\tau}\right)(\omega) \| \leq 1 / 2^{k}$. For every $k \geq 1$, every positive $\delta \leq \delta_{k}$ we have

$$
\sup _{t_{0} \leq t \leq t_{0}+\delta}\left\|\int_{t_{0}}^{t} \mathcal{G}^{p} d B_{\tau}\right\| \leq \sup _{t_{0} \leq t \leq t_{0}+\delta_{k}}\left\|\int_{t_{0}}^{t} \mathcal{G}^{p} d B_{\tau}\right\|,
$$

which implies that for a.e. $\omega \in \Omega$ there is $n(\omega) \geq 1$ such that for every $k \geq n(\omega)$ there is $\delta_{k}>0$ such that for every $\delta \in\left(0, \delta_{k}\right]$ we have $\left.\sup _{t_{0} \leq t \leq t_{0}+\delta} \| \int_{t_{0}}^{t} \mathcal{G}^{p} d B_{\tau}\right)(\omega) \|$ $\leq 1 / 2^{k}$. Then

$$
\lim _{\delta \rightarrow 0} \sup _{t_{0} \leq t \leq t_{0}+\delta} h\left[\left(\int_{0}^{t} \mathcal{G}^{p} d B_{\tau}\right)(\omega),\left(\int_{0}^{t_{0}} \mathcal{G}^{p} d B_{\tau}\right)(\omega)\right]=0
$$

for a.e. $\omega \in \Omega$. In a similar way we obtain

$$
\lim _{\delta \rightarrow 0} \sup _{t_{0}-\delta \leq t \leq t_{0}} h\left[\left(\int_{0}^{t} \mathcal{G}^{p} d B_{\tau}\right)(\omega),\left(\int_{0}^{t_{0}} \mathcal{G}^{p} d B_{\tau}\right)(\omega)\right]=0
$$

for a.e. $\omega \in \Omega$, with $0<\delta<t_{0}$ for every $t_{0}>0$. Thus the set-valued stochastic process $\left(\int_{0}^{t} \mathcal{G}^{p} d B_{\tau}\right)_{t \geq 0}$ is continuous.

Remark 5.1. In a similar way we can prove that for a given $m$-dimensional $\mathbb{F}$-Brownian motion $B=\left(B_{t}\right)_{t \geq 0}$ defined on $\mathcal{P}_{\mathbb{F}}$ and every sequence $\left(g^{n}\right)_{n=1}^{\infty} \subset$ $\mathbb{L}^{2}\left(\mathbb{R}^{+} \times \Omega, \Sigma_{\mathbb{F}}, \mathbb{R}^{d \times m}\right)$ such that $\sum_{n=1}^{\infty}\left\|g^{n}\right\|^{2}<\infty$, a set-valued stochastic pro$\operatorname{cess}\left(\int_{0}^{t} \mathcal{G} d B_{\tau}\right)_{t \geq 0}$ defined by the set $\mathcal{G}=\operatorname{co}\left\{g^{n}: n \geq 1\right\}$, is a continuous set-valued submartingale.

Remark 5.2. Because of unboundedness of set-valued stochastic integrals, defined in the paper [4], we can not consider stochastic differential equations $x_{t}=x_{0}+\int_{0}^{t} F\left(\tau, x_{\tau}\right) d \tau+\int_{0}^{t} G\left(\tau, x_{\tau}\right) d B_{\tau}$ with set-valued solutions in the general case, i.e., if $G$ is not a singleton. Instead of, we can consider stochastic differential equation of the form $x_{t}=x_{0}+\int_{0}^{t} F\left(\tau, x_{\tau}\right) d \tau+\int_{0}^{t} \mathcal{G}_{G}(x) d B_{\tau}$, where $\mathcal{G}_{G}(x)=\operatorname{co}\{g \circ x: g \in \mathcal{G}\}$ for every nonempty set $\mathcal{G}$ of Carathèodory selectors of $G$ such that a set-valued stochastic integral $\int_{0}^{t} \mathcal{G}_{G}(x) d B_{\tau}$ is square integrably bounded and $(g \circ x)_{t}(\omega)=g\left(t, x_{t}(\omega)\right)$ for every $(t, \omega) \in \mathbb{R}^{+} \times \Omega$. 


\section{REFERENCES}

[1] F. Hiai and H. Umegaki, Integrals, conditional expectations, and martingales of multivalued functions, J. Multivariate Anal. 7 (1977) 149-182. doi:10.1016/0047-259X(77)90037-9

[2] W. Hildenbrand, Core and Equilibria of a Large Economy (Princeton University Press, 1974).

[3] Sh. Hu and N.S. Papageorgiou, Handbook of Multivalued Analysis I, (Kluwer Academic Publishers, 1997). doi:10.1007/978-1-4615-6359-4

[4] E.J. Jung and J.H. Kim, On the set-valued stochastic integrals, Stoch. Anal. Appl. 21 (2)(2003) 401-418. doi:10.1081/SAP-120019292

[5] M. Kisielewicz, Viability theorems for stochastic inclusions, Discuss. Math. 15 (1995) 61-74.

[6] M. Kisielewicz, Set-valued stochastic integrals and stochastic inclusions, Stoch. Anal. Appl. 15 (5) (1997) 783-800. doi:10.1080/07362999708809507

[7] M. Kisielewicz, Some properties of set-valued stochastic integrals, J. Math. Anal. Appl. 388 (2012) 984-995. doi:10.1016/j.jmaa.2011.10.050

[8] M. Kisielewicz, Stochastic Differential Inclusions and Applications (Springer, New York, 2013). doi:10.1007/978-1-4614-6756-4

[9] M. Kisielewicz, Some properties of set-valued stochastic integrals of multiprocesses with finite Castaing representations, Comm. Math. 53 (2) (2013) 213-226.

[10] M. Kisielewicz, Martingale representation theorem for set-valued martingales, J. Math. Anal. Appl. 409 (2014) 111-118. doi:10.1016/j.jmaa.2013.06.066

[11] M. Michta, Remarks on unboundedness of set-valued Itô stochastic integrals, J. Math. Anal. Appl. (presented to print). 
